\title{
Toward Optimization of Rheology in Sea Ice Models through Data Assimilation
}

\author{
J. N. STROH \\ Department of Atmospheric Science, University of Alaska Fairbanks, Fairbanks, Alaska \\ Gleb PanteleEV AND MaX YAREMchuK \\ Naval Research Laboratory, Stennis Space Center, Mississippi \\ OCEANA FRANCIS \\ Department of Civil and Environmental Engineering, University of Hawai'i at Mānoa, Honolulu, Hawaii \\ RICHARD ALLARD \\ Naval Research Laboratory, Stennis Space Center, Mississippi
}

(Manuscript received 23 December 2018, in final form 27 June 2019)

\begin{abstract}
Sea ice models that allow for deformation are primarily based on rheological formulations originally developed in the 1970s. In both the original viscoplastic (VP) and elastic-VP schemes, the internal pressure term is modeled as a function of variable sea ice thickness and concentration with spatially and temporally constant empirical parameters for ice strength. This work considers a spatially variable extension of the rheology parameters as well as wind stress in a one-dimensional VP sea ice data assimilation system. In regions of total ice cover, experiments that assimilate synthetic ice-state observations using variable rheological parameters show larger improvements than equivalent experiments using homogeneous parameters. For partially ice-covered regions where internal ice stresses are relatively unimportant, experiments assimilating synthetic sea ice velocity observations demonstrate reasonable reconstruction of spatially variable wind stresses. These results suggest practical benefits for sea ice-state reconstruction and forecasts by using sea ice velocity, thickness, and concentration observations to optimize spatially varying rheological parameters and to improve wind stress forcing.
\end{abstract}

\section{Introduction}

Sea ice models are an important component of any ice-ocean data assimilation (DA) system in the Arctic Ocean (AO) and the Southern Ocean. Currently, there are several DA systems that are widely applied to reconstruct Arctic ice conditions in reanalysis or quasioperational mode. For example, there are systems based on the MITgcm (Menemenlis et al. 2008; Heimbach 2008; Forget et al. 2015; Fenty et al. 2017), ROMS (Wang et al. 2013), HYCOM (Lisæter et al. 2007; Sakov et al. 2012), Pan-Arctic Ice Ocean Modeling and Assimilation System (PIOMAS; Zhang and Rothrock 2003; Lindsay and

\footnotetext{
${ }^{\text {a }}$ Current affiliation: Department of Bioengineering, University of Colorado Denver, Aurora, Colorado.
}

Corresponding author: J. N. Stroh, jnstroh@alaska.edu
Zhang 2006), and NEMO (Vancoppenolle et al. 2009; Massonnet et al. 2015). Several new methods of sea ice modeling have been proposed during the last decade including Lagrangian models (Rampal et al. 2016; Bouillon and Rampal 2015b) and finite element models (Danilov et al. 2015). In spite of these new technologies, practically all ice-ocean DA systems implement sea ice models, such as the Los Alamos sea ice model (CICE; Hunke et al. 2010), which are based on the viscoplastic (VP) rheology proposed by Hibler (1979) or its associated elasto-VP (EVP) numerical scheme (Hunke and Dukowicz 1997). The essential advantage of these models is the efficient and relatively simple approximation of sea ice dynamics in terms of the VP/EVP rheology that is based on a rigorous theoretical framework developed in the past century (Timoshenko and Goodier 1951; Goodier and Hodge 1958) with an adjunct practical stability analysis theory for sea ice 
applications (Gray and Killworth 1995; Schulkes 1996; Dukowicz 1997; Pritchard 2005).

According to Hibler (1979), the sea ice model rheology is defined by three parameters $e, P^{*}$, and $c^{*}$ describing, respectively, the yield ellipse eccentricity, the dimensional maximum ice strength per unit thickness, and nondimensional scaling of ice strength with its compactness. Ice strength within a grid cell is a mean value, although the intracell strength distribution is unknown. The VP rheology approximates sea ice dynamics by statistically representing spatiotemporal variations in plastic deformation rates as a viscosity term (Hibler 1977). Therefore, practical VP viscosity reflects a subgrid parameterization of variable local strength properties without resolving the underlying floe distribution. However, one notes that this interpretation implicitly presumes a uniform distribution of cracks and other ice weaknesses throughout the grid cell.

Modeled sea ice results are sensitive to values of sea ice rheological parameters (RPs) (Massonnet et al. 2011), making their estimation in models important for the reconstruction and forecast of ice states. RPs such as $P^{*}$ and $e$ reflect the model parameterization rather than physics and are not directly observable (Kreyscher et al. 2000), but nevertheless are known to range within limits (Harder and Fischer 1999). For example, VP model values of $P^{*}$ determined by optimizing sea ice drift include $27.5 \mathrm{kN} \mathrm{m}^{-2}$ (Hibler and Walsh 1982), $15-20 \mathrm{kN} \mathrm{m}^{-2}$ (Kreyscher et al. 1997, 2000), and $30-45 \mathrm{kN} \mathrm{m}^{-2}$ (Tremblay and Hakakian 2006). The latter work emphasizes the significant variability of their $P^{*}$ estimates, which may be attributed to spatial and temporal variation of Arctic sea ice and better represented using a nonelliptic form of the yield curve (e.g., Tremblay and Hakakian 2006). Recently, the impact of spatial nonuniformity of the ice strength parameter $P^{*}$ was analyzed by Juricke et al. (2013). AO sea ice models in that study generated greater ice volume and lower ice cover area when $P^{*}$ was stochastically perturbed, and the authors found that the effects of such spatiotemporal parameter variability could not be reproduced by constant global modifications of $P^{*}$.

For modeling landfast ice, an additional parameter $k_{T}$ has been introduced to account for tensile strength (König Beatty and Holland 2010) that is omitted in the traditional (i.e., Hibler 1979) elliptical yield curve formulation. Lemieux et al. (2016) used numerical experiments with a coarse-resolution pan-Arctic CICENEMO model to find a tensile ratio value $k_{T}=0.2$ and ellipse ratios in the range $1.2 \leq e \leq 1.4$ provide the best agreement with landfast ice distribution observations in the Kara Sea region. The work notes that "tensile strength should depend on other factors such as sea ice salinity, the ice temperature, the history of deformation of sea ice, etc." and intimates the possible variable nature of $e$ due to the significant impact of ellipse parameter values on simulation results. Recent work by Toyota and Kimura (2018) found optimal VP rheology parameterization with $e$ centered in the range of 1.7-2.0, noting dependence of the optima on both model resolution and region.

Thus, analysis of sea ice observations and numerical simulations suggest that an optimized spatially varying set of RPs [i.e., $e(x, y), P^{*}(x, y), c^{*}(x, y), k_{T}(x, y)$ ] may significantly improve the results of numerically modeled sea ice. One also anticipates the time dependence of RPs due to the effects of ice history and seasonal variability in the surrounding environment. It is tempting to methodically resolve RP dependence on observable sea ice properties and analytically derive optimal estimates from them. However, a poor theoretical understanding of ice floe interaction subject to various external and internal forces deters this approach.

An alternative method is to apply a variational data assimilation system (VarDAS) employing the methods of Le Dimet and Talagrand (1986) to empirically optimize model RPs within a sea ice model on the basis of assimilated sea ice observations. This approach looks feasible given the tremendous increase of the satellite sea ice observations during the last decades suitable for use as model constraints. When ice cover is not full, the permitted advection exponentially reduces the importance of internal stresses so the benefit of variable RPs may be relatively unimportant for sea ice model dynamics in this case. In such regions, however, model dynamics may benefit from optimization of windimparted surface stresses (Miller et al. 2006) that depend on the local ice state (Steele et al. 1989; Steiner 2001). A common assimilation approach can be generally applied to find optimal RP and/or wind stress depending on the region.

There are currently many sources of observational data for sea ice, including relatively accurate observations of sea ice concentration (SIC) and sea ice velocity (SIV) from optical or synthetic aperture radar (SAR) images (Komarov and Barber 2014; Bouillon and Rampal 2015a). Several satellite projects may potentially provide daily SIV and sea ice thickness (SIT) in the near future [e.g., NASA ICESat-2 and the Canadian Space Agency (CSA) RadarSat Constellation Mission] given the right data processing. A properly developed variational algorithm can assimilate these sea ice observations to optimize spatially variable sea ice RPs in the Arctic region.

This study demonstrates that spatially varying parameters in an assimilative sea ice model strongly improve the quality of observationally optimized model trajectories. It addresses the topic of controllability of 
VP ice models through sea ice RPs, which is worthwhile to explore as a first step toward extending this method to modern sea ice models (e.g., CICE) by developing the associated adjoint code needed for variational assimilation. Static RP distributions are sought here for the purposes of improving short-term reconstruction and forecast of the ice state, although temporal variability may be resolved by sequential application of the assimilation technique.

The presented manuscript adopts the simplified onedimensional sea ice model of König Beatty and Holland (2010) and explores some of these questions using the variational assimilation method. The developed VarDAS may be formally applied to estimate sea ice rheology in narrow straits or small-scale regions where sea ice is essentially one-dimensional such as in ice ridging areas. Observation system simulation experiments (OSSEs) evaluate the quality of optimized solutions achieved through control of two major sea ice RPs ( $P^{*}$ and $e$ ), wind stress, and initial ice-state distributions on the basis of simulated noisy satellite observations.

The paper is organized as follows: section 2 describes the implemented sea ice model, applied DA algorithm, and observational data; section 3 describes the underlying OSSE hypotheses and experiment design; section 4 discusses results of the OSSEs; and section 5 summarizes the work and comments on future research.

\section{Optimization method}

This section details the implemented forward-time sea ice model and its associated linearizations, outlines the variational data assimilation system for optimizing model parameters and initial ice state, and describes the synthetic observations used to do so.

\section{a. Forward, tangent linear, and adjoint models}

This work considers the thermostatic sea ice model (Hibler 1979) with tensile strength (König Beatty and Holland 2010) and localized variability in ice-strength parameters. The standard one-dimensional sea ice constituent equations with no-slip boundaries on a length $L$ domain are given by

$$
\begin{aligned}
m \frac{\partial u}{\partial t} & =\frac{\partial \sigma}{\partial x}+\tau_{a}(x)-\tau_{o}, \quad u(x, 0)=u_{0}(x), \\
\frac{\partial h}{\partial t} & =-\frac{\partial(u h)}{\partial x}, \quad h(x, 0)=h_{0}(x), \\
\frac{\partial a}{\partial t} & =-\frac{\partial(u a)}{\partial x}, \quad a(x, 0)=a_{0}(x),
\end{aligned}
$$

with a stress tensor defined by

$$
\begin{aligned}
\sigma & =\frac{e(x)^{2}+1}{e(x)^{2}} \frac{P+T}{2 \Delta} \frac{\partial u}{\partial x}-\frac{P-T}{2}, \\
P & =P^{*}(x) h \exp \left[-c^{*}(x)(1-a)\right], \\
T & =k_{T}(x) P, \\
\Delta & =\max \left\{\Delta_{\min },\left|\frac{\partial u}{\partial x}\right| \sqrt{\frac{e(x)^{2}+1}{e(x)^{2}}}\right\},
\end{aligned}
$$

where $e(x), k_{T}(x), P^{*}(x)$, and $c^{*}(x)$ are spatially (piecewise) variable distributions of the ellipse ratio, tensile strength factor, and empirical ice-strength parameters, respectively; the symbol $\Delta_{\text {min }}$ denotes the creep limit; $u(x, t), h(x, t)$, and $a(x, t)$ identify SIV, SIT, and SIC, respectively; and $\tau_{a}(x)$ and $\tau_{o}$ denote respective atmospheric and oceanic stresses.

The general form of surface stress is given by $\tau_{i}=$ $C_{i} a\left|u_{i}-u\right|\left(u_{i}-u\right)$ where the subscript $i$ is either $a$ for atmospheric or $o$ for oceanic sources, with $u_{i}$ and $C_{i}$, respectively, denoting the associated velocity and drag coefficient of the source. For simplicity, omission of the ocean stress and the impact of sea ice velocity on the wind stress reduces the external forcing to a wind stress of the form $\tau_{a}(x)=C_{a} a(x)\left|u_{a}(x)\right| u_{a}(x)$ where $u_{a}$ is the $10-\mathrm{m}$ wind velocity. In regions with low sea ice concentration $(a<0.8)$, neglect of the ocean drag may result in the gradual increase of SIV and consequent violation of the numerical CFL condition. However, this was not problematic in the relatively short periods of model integration considered here. The model imposes timeinvariant (constant) boundary conditions on thickness and concentration, and nonslip (zero velocity) boundary conditions on ice velocity. Initial ice conditions implicitly determine the fixed boundary values for SIT and SIC. The strict type of boundary conditions used here are easily relaxed in the model, but doing so increases the computational cost of model control. Note that this model also omits the dynamical influences of sea surface tilt and Coriolis effects.

The 1D rheology parameterization nonuniquely represents compressive ice strength $P$ and net pressure factor $P-T$ in terms of $\left(P^{*}, c^{*}\right)$ and $\left(P, k_{T}\right)$, respectively. Therefore, variability of the pair $\left[P^{*}(x), e(x)\right]$ is sufficient to fully parameterize the $1 \mathrm{D}$ rheology, and invariant values are set for $c^{*}=20$ and $k_{T}=0$ as in Table 1. Omission of $c^{*}(x)$ and $k_{T}(x)$ from the control results in equivalent controllability with reduced control vector dimension, although the modeling system retains variability of these variables for future $2 \mathrm{D}$ development where this is not true. With these assumptions in place, the stress term of Eq. (4) simplifies to 
TABLE 1. Model and assimilation system configuration parameters.

\begin{tabular}{lcc}
\hline \multicolumn{1}{c}{ Name } & Symbol & Value(s) \\
\hline Domain size & $L$ & $1800 \mathrm{~km}$ \\
Grid size & $d x$ & $10 \mathrm{~km}$ \\
Time step & $d t$ & $600 \mathrm{~s}$ \\
Integration time & $T$ & $5.5 \mathrm{days}$ \\
Assimilation window & $\tau_{o}$ & $0 \leq t<4$ days \\
Ice-ocean stress & $\Delta_{\min }$ & 0 \\
Creep limit & $u_{a}$ & $0.1 \mathrm{~s}^{-1}$ \\
Background wind speed & $c^{*}$ & $20 \cos (x / L-1 / 2) \mathrm{m} \mathrm{s}^{-1}$ \\
Compactness strength parameter & $k_{T}$ & 20 \\
Tensile/compressive strength ratio & $P^{*}(x)$ & 0 \\
Base strength parameter & $e(x)$ & {$[22,35] \mathrm{kN} \mathrm{m}^{-2}$} \\
Yield curve eccentricity & $C_{a}$ & {$[1.5,2.5]$} \\
Atmospheric drag coefficient & {$[u(0, t), h(0, t), a(0, t)]$} & $0.0013 \mathrm{~kg} \mathrm{~m}{ }^{-3}$ \\
Fixed left boundary values & {$[u(L, t), h(L, t), a(L, t)]$} & {$\left[0, h_{0}(0), a_{0}(0)\right]$} \\
Fixed right boundary values & {$\left[0, h_{0}(L), a_{0}(L)\right]$}
\end{tabular}

$$
\sigma(x)=\left[\frac{e(x)^{2}+1}{e(x)^{2}} \frac{\partial u / \partial x}{\Delta}-1\right] \frac{P(x)}{2}
$$

Discretization of Eqs. (1) and (5)-(8) over a B-grid at time step with index $k$ yields a tridiagonal matrix equation

$$
\mathbf{A}_{k}\left(\mathbf{u}^{k}, \mathbf{h}^{k}, \mathbf{a}^{k}\right) \mathbf{u}^{k+1}=\mathbf{b}^{k}
$$

(König Beatty and Holland 2010), defining an implicit map from currently known state variables and parameters $\mathbf{b}^{k}$ to forecast velocities $\mathbf{u}^{k+1}$. The map $\mathbf{A}_{k}$ is clearly nonlinear as it depends on the current SIV, SIT, and SIC distributions $\mathbf{u}^{k}, \mathbf{h}^{k}$, and $\mathbf{a}^{k}$, respectively. Equivalently discretized continuity equations [Eqs. (2) and (3)] update concentration and thickness once velocities at time $k+1$ are known. These equations augment Eq. (9) to give an implicit full update of the model sea ice state, and Eq. (9) hereafter refers to this augmented system.

The model was configured for a $L=1800-\mathrm{km}$ domain discretized with $10-\mathrm{km}$ resolution and forced by stationary but spatially varied wind for 5.5 days. Stationarity of the wind stress over the integration period only influences the model dynamics, but is not a factor in optimizability; the linear dynamical response to atmospheric stress in Eq. (1) implies that the optimization depends only on the wind stress errors rather than its background state at each time step. For testing and experimentation presented here, atmospheric winds and initial sea ice conditions were specified analytically by combinations of trigonometric functions, while RPs were specified by piecewise linear functions. This approximation significantly decreases the dimension of the control vector involved in the inverse problem discussed below. Other numerical model parameters are largely adopted from König Beatty and Holland (2010) and assembled in Table 1.

Equation (9) is solved numerically using generalized minimum residual (GMRES) routines (Saad and Schultz 1986) as suggested by Lemieux et al. (2008). Exact tangent linear and adjoint models associated with Eq. (9) were developed analytically with respect to a control vector comprising initial-state distributions $\left[u_{0}(x), a_{0}(x)\right.$, $h_{0}(x)$ ], wind stress $\tau_{a}(x)$, and RP vectors $P^{*}(x), e(x)$, $c^{*}(x)$, and $k_{T}(x)$. The adjoint system is solved using a conjugate gradient method with bounded constraints, which restricts feasible controls to realistic ranges through the inequalities $22000 \leq P^{*}(x) \leq 35000,1.5 \leq e(x) \leq 2.5$, $0 \leq a_{0}(x) \leq 1$, and $0.2 \leq h_{0}(x) \leq 6.0$. These RP constraints suffice for experimental purposes where a reference value is specified within them; they are easily widened appropriately for practical application. Solutions to the nonlinear and adjoint systems are used to determine an optimal control vector on the basis of observations using the VarDAS described below.

\section{b. Variational assimilation method}

The update of the sea ice model state implied by Eq. (9) together with discretized continuity equations define a numerical forward-time state map. By iterating this function, model states depend only on the initialstate conditions and static model parameters that compose the control vector: $\mathbf{x}=\mathfrak{M}(\mathbf{c})$ where $\mathbf{x}$ denotes the trajectory of model ice variables $u(x, t), h(x, t)$, and $a(x, t) ; \mathfrak{M}$ is the iterated forward-time map; and $\mathbf{c}$ is a control vector composed of complete initial-state conditions along with other model parameters. The associated tangent linear model $\delta \mathbf{x}=\mathbf{M} \delta \mathbf{c}$ identifies variations of the model state $\delta \mathbf{x}$ with respect to control vector variations $\delta \mathbf{c}$; the adjoint model, $\delta \mathbf{c}=\mathbf{M}^{\mathrm{T}} \delta \mathbf{x}$, where $(\cdot)^{\mathrm{T}}$ is the matrix transpose, identifies the control variations 
associated with a change in state. The optimal control vector is sought to ensure that observations of model states $\{\mathfrak{h}(\mathbf{x})\}$ lie close to known observations $\{\mathbf{y}\}$ by minimizing the standard quadratic variational cost function $J(\mathbf{c})$ (Le Dimet and Talagrand 1986) in two dimensions. This unknown minimum is distinguished by the vanishing gradient of $J$ at the optimum control $\mathbf{c}_{\text {opt }}$, and may be identified from a first-guess control $\mathbf{c}_{0}$ by solving

$$
0=\mathbf{B}^{-1} \mathbf{M} \delta \mathbf{c}+\sum_{k=0}^{N} \mathbf{M}_{k}^{\mathrm{T}} \mathbf{H}_{k}^{\mathrm{T}} \mathbf{R}^{-1}\left(\mathbf{H}_{k} \mathbf{M}_{k} \delta \mathbf{c}-\mathbf{d}^{k}\right),
$$

where $\mathbf{B}$ and $\mathbf{R}$ are the background model and observation error covariance matrices, respectively; $0 \leq k \leq N$ are model time indices of the assimilation window; $\mathbf{H}_{k}$ denotes the linearization of the observation operator $\mathfrak{h}$ at time $k$; and $\mathbf{d}^{k}=\mathbf{y}^{k}-\mathfrak{h}\left[\mathfrak{M}\left(\mathbf{c}_{0}\right)_{k}\right]$ is the first-guess difference from observation at time $k$. The symbol $\mathbf{M}_{k}$ denotes application of the tangent linear model from the initial time to time $k$, and $\mathbf{M}_{k}^{\mathrm{T}}$ identifies the adjoint model at time $k$. Error covariances in this study are diagonal with entries defined by component-wise estimates of appropriate variances. The optimal control $\mathbf{c}_{\mathrm{opt}}=\mathbf{c}_{0}+$ $\delta \mathbf{c}_{\text {opt }}$ contains optimal initial-state conditions and RP distributions reported in the following sections; the trajectory of ice model states $\mathfrak{M}\left(\mathbf{c}_{\mathrm{opt}}\right)$ is reported hereafter as the optimal model solution. Components of the control may be readily omitted from optimization in experiments by preventing their update to formally retain their presence in c. Preliminary VarDAS experiments determined that the most effective strategy for minimizing $J$ in this case was to first optimize the ice model initial conditions, followed by optimization of the remaining control components $P^{*}(x), e(x)$, and $\tau_{a}(x)$ together.

\section{c. Observations}

For experimental purposes, observational data of the sea ice state are required for assimilation. This work aims to represent synthetic equivalents of the following high-resolution satellite observations, which are currently available or developing.

The first data are accurate SIC observations, of which there are currently multiple gridded products based on various remote sensing instruments with different spatial resolutions. Example datasets include the Passive Microwave Sea Ice Concentration with $25-\mathrm{km}$ resolution (Peng et al. 2013; https://nsidc.org/data/g02202), the Advanced Microwave Scanning Radiometer 2 (AMSR2) product with $10-\mathrm{km}$ resolution (https://gportal.jaxa.jp/), and the Multisensor Analyzed Sea Ice Extent (MASIE) AMSR2 (MASAM2) analysis with 4-km resolution (https:// nsidc.org/data/g10005). After additional preprocessing, these observations are routinely used in data assimilation systems [e.g., Global Ocean Forecast System (GOFS) 3.1 DA system; Cummings and Smedstad 2013] with a nominal spatial resolution of $5 \mathrm{~km}$ and regionally low SIC representation errors ( $\sim \%$; Yaremchuk et al. 2019).

The second set are SIT observations that contain moderate errors. Currently, the primary source of such data is CryoSat-2, with 1- and 5-km gridded 2-dayaveraged observations available from the Center for Polar Observations and Modeling (http://www.cpom.ucl.ac.uk/ csopr/seaice.html). The process of converting observed freeboard height to equivalent sea ice thickness strongly depends on other ice properties such as the age of the ice and surface snow depth (Alexandrov et al. 2010); recent works establish CryoSat-2 inaccuracies of $0.46-0.74 \mathrm{~m}$ (Alexandrov et al. 2010), $0.624 \mathrm{~cm}$ (Laxon et al. 2013), and $0.34-0.66 \mathrm{~m}$ (Tilling et al. 2018). In the future, novel observational platforms and methods of analysis will likely provide better spatial coverage (i.e., over the entire Arctic) and improved accuracy. Experiments in this work posit future SIT observation standard errors of 0.25 and $0.35 \mathrm{~m}$ in the pack ice zone (PIZ) and marginal ice zone (MIZ), respectively.

Accurate observations of sea ice velocities compose the third group. An example data product is the daily $25-\mathrm{km}$ SIV analysis of various satellite and in situ sources (Tschudi et al. 2019) whose uncertainty was established at 0.01-0.02 $\mathrm{m} \mathrm{s}^{-1}$ (Schwegmann et al. 2011; Sumata et al. 2015) while product documentation reports standard errors of about $0.033 \mathrm{~m} \mathrm{~s}^{-1}$. However, new methods of sequential SAR image comparison can resolve high-resolution SIV with accuracy of $\sim 0.005 \mathrm{~m} \mathrm{~s}^{-1}$ (Komarov and Barber 2014), which suggests the possibility of high-precision SIV observations in the near future. The estimated inaccuracy of SIV is assumed to be $0.025 \mathrm{~m} \mathrm{~s}^{-1}$ to reflect the current accuracy of available gridded data.

A fourth set of data is needed to optimize atmospheric forcing and comes in the form of wind stress information. Atmospheric wind stress is usually calculated from the 10-m wind field of atmospheric reanalysis or weather forecast systems, rather than observations. The inaccuracy of such data in the central Arctic region likely results from limited in situ observations for calibration rather than inaccuracies in the atmospheric boundary layer parameterization of the existing models. Evaluation of product accuracy by Jakobson et al. (2012) suggests that standard wind speed errors are typically $1.5 \mathrm{~m} \mathrm{~s}^{-1}$ in the central $\mathrm{AO}$; the corresponding wind stress uncertainty calculated at full ice cover using parameters in Table 1 is about $0.04 \mathrm{~N} \mathrm{~m}^{-2}$ and scales linearly with open water fraction. However, this estimate fails to account for uncertainties in the static ice-air 


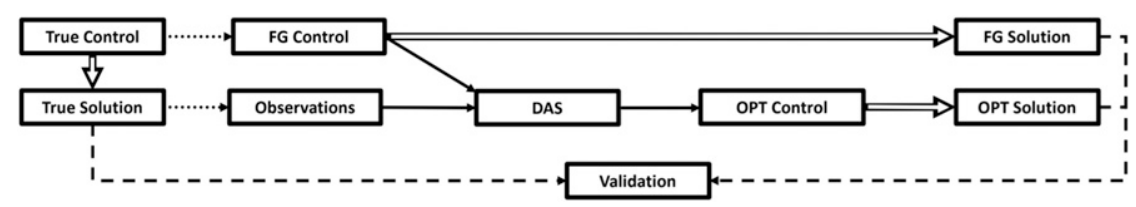

FIG. 1. Schematic of an OSSE. The general process of an OSSE is to generate noisy observations from an a priori natural solution, to assimilate/not assimilate those data into an inaccurately initialized model, and to compare the resulting solutions with the true one. Dotted arrows correspond to noisy processes, and hollow arrows correspond to application of the forward model. Unbroken arrows correspond to system input/output, and the dashed arrows identify the quantities compared in the experiment validation.

friction coefficient and errors in SIC estimates. Also, atmospheric products such as NASA Modern-Era Retrospective Analysis for Research and Applications (MERRA; Cullather and Bosilovich 2011) and ECMWF interim reanalysis (ERA-Interim; Dee et al. 2011) with resolutions of 50 and $80 \mathrm{~km}$, respectively, incur considerable representation errors when transferred to a much finer model grid. Further, the 1D wind stress discussed here lacks a vorticity component that must be accounted for in realistic 2D applications. For these reasons, the uncertainties associated with $1 \mathrm{D}$ wind stress data are assumed to be much larger with a standard error of $0.125 \mathrm{~N} \mathrm{~m}^{-2}$ in the PIZ and reduced to $0.04 \mathrm{~N} \mathrm{~m}^{-2}$ in the MIZ as discussed later.

This work assumes that spatial resolution of synthetic observation representatives is the same as the model resolution, and also that observations are available at each 10-min model time step. Practical applications justify spatiotemporal interpolation of existing sea ice datasets because they are already gridded, daily analyses of asynchronous satellite swaths. Here, assumptions are motivated by simplicity and the desire to avoid specifying additional smoothing terms needed to regularize the ill-posed optimization problem (i.e., Neumaier 1998, and references therein).

\section{Observing system simulation experiments}

The sea ice model presented in Eqs. (1)-(7) suggests two major dynamical regimes depending on ice coverage. This grouping motivates the organization of experiments as their dynamics are distinct.

The first regime occurs where sea ice covers the entire ocean surface; it is identified as the PIZ and characterized by $a(x) \approx 1$. Within this case, internal stress $\sigma$ plays a significant role in the sea ice dynamics and SIC continuity [Eq. (3)] may be ignored by assuming $a(x)=1$. Formally, this assumption of a constant, full ice cover is equivalent to immediate sea ice formation in regions where SIC slightly decreases due to ice divergence. Equation (4) naturally suggests that spatial variability of RPs should significantly affect the ice dynamics in convergent PIZ regions such as influencing the formation of ice ridges.

The second regime occurs where sea ice is present but only partially covers the ocean surface; it is identified as the MIZ and characterized by concentrations $0.2 \leq$ $a(x) \leq 0.8$ in this work. Numerically, this case differs from that of the PIZ because Eq. (3) is required to solve Eq. (9). In the MIZ, the impact of the internal stress $\sigma$ is low as ice movement is not fully constrained. Since surface tilt is ignored, dynamics of the presented sea ice model in this regime are controlled by external forcing, together with Coriolis effects in the 2D case.

Wind stress is naturally the most significant source of external forcing in this ice model, and accuracy of the wind forcing is crucial to securing quality reconstruction and forecasts of the sea ice state. It is thus reasonable to conduct OSSEs for cases that involve idealized, errorfree atmospheric forcing along with cases using realistic atmospheric forcing that reflects the limitations and uncertainties of the source products. Similarly, one should consider the impact of optimization with respect to the various control components: the initial ice state $\left[u_{0}(x)\right.$, $\left.a_{0}(x), h_{0}(x)\right]$, the wind stress $\tau_{a}(x)$, and the RP distributions $\left[P^{*}(x), e(x)\right]$.

To assess the quality of optimized sea ice model solutions using various controls and data sources, OSSEs are conducted in which poorly initialized model results are compared with identically initialized VarDAS solutions. The conventional OSSE methodology (Houtekamer and Mitchell 1998) is briefly described here (see Fig. 1).

First, "true" solutions are generated with a given atmospheric forcing, initial-state conditions, and RP distributions. Initial SIT and SIC conditions are specified as the sum of several smooth cosine and sine functions. Initial SIV conditions are determined by a 10-time-step (100 $\mathrm{min}$ ) forward model integration starting from a rest state with all other initial variables and parameters the same. Synthetic observational data are generated by contaminating this true, known solution with spatially correlated noise whose amplitude and scale depends on the type of observation the data is intended to represent. 
The model is then reinitialized with inaccurate firstguess (FG in figures) initial conditions, wind stress, and $\mathrm{RP}$ that compose the first-guess control $\mathbf{c}_{0}$. In all experiments presented, first-guess control includes constant RP distributions to mimic the conventional approach. The variational assimilation scheme presented in section $2 b$ is then applied with perfect model assumptions to determine the optimal control $\mathbf{c}_{\text {opt }}$ by assimilating observations during the interval $0 \leq t<4$ days. The optimal model state trajectory and parameters (OPT in figures) result from initialization at the start of day 0.0 .

Noisy observations are generated by adding a stationary random signal to each state variable of the true solution at each time step, and are intended to mimic realistic observations such as those obtained from previously mentioned sources and interpolated to the sea ice model grid. The wind stress observations are treated similarly, although its true state remains constant in time. Magnitudes of the imposed noise correspond to the estimated errors for the observational data discussed in section $2 \mathrm{c}$, with amplitudes of $5 \%, 0.25-0.35 \mathrm{~m}, 0.025 \mathrm{~m} \mathrm{~s}^{-1}$, and $0.04-0.125 \mathrm{~N} \mathrm{~m}^{-2}$ for SIC, SIT, SIV, and wind stress, respectively. The noise is then smoothed by applying a cosine function to provide approximate decorrelation length scales of $50-65 \mathrm{~km}$ for state variables and $190 \mathrm{~km}$ for wind stress, respectively. This modulation of the random errors to impose a spatial scale affects the mean error of the applied noise, and the mean resulting amplitude of these corruptions is reported hereafter. All assimilated observations include noise of this form and their error structure is common among the experiments. However, MIZ OSSEs feature increased SIT errors and decreased SIV errors as noted.

Initial conditions for the first-guess control are generated similarly with decorrelation length scales of 250$350 \mathrm{~km}$ for SIT and SIC ice variables; this increased scale simulates an initialization state obtained from a previous observation-conditioned model. This longer spatial scale also supports an initial ice state whose trajectory evolves naturally away from the true solution. In contrast, initial perturbations with a shorter spatial scale are often dissipated and smoothed by the model dynamics without strongly affecting state variable evolution. The initial SIV is again specified via 100 min of integration from a motionless state using the other first-guess initial conditions.

In discussing experimental results, it is often necessary to distinguish between the control vector and the trajectory (or solution) resulting from it. For example, a first-guess control $\mathbf{c}_{0}$ includes initial conditions for SIV, SIT, and SIC at $t=0$ as well as parameter distributions of RPs and $\tau_{a}(x)$; application of the forward model results in the first-guess solution $\mathbf{x}_{0}=\mathfrak{M}\left(\mathbf{c}_{0}\right)$. The true and optimal solutions are defined analogously by the initialstate conditions and parameters composing their associated control vectors.

Each experiment consist of two separate stages: a hindcast period $(0 \leq t<4$ days) during which data assimilation occurs in the optimized model run, and a forecast period ( $4.0 \leq t \leq 5.5$ days) during which all models are unconstrained. The quality of reconstruction based on the assimilated data is assessed during the former period, while the corresponding improvement in predictability is measured during the latter. Skill of the model runs is calculated separately for each experiment stage by the root-mean-square error (RMSE) of simulated quantities $\left[u(x, t), a(x, t), h(x, t), \tau_{a}(x)\right]$ with respect to their true counterparts. The RMSEs reported for ice variables correspond to the innermost $1660 \mathrm{~km}$ of the domain, averaged over all time steps of the hindcast and forecast periods independently; the boundary regions are discarded here to omit errors likely caused by dynamical imbalance between forcing and boundary conditions. Wind stress errors are reported for the entire length of the domain. Conducted OSSEs are detailed concisely in Table 2.

\section{Results and discussion}

\section{a. Pack ice zone}

Figure 2 (left) shows the true solution associated with PIZ experiments (OSSE-3, OSSE-3w, and OSSE- $3 n$ ). The true parameters are piecewise linear distributions for $P^{*}(x)$ and $e(x)$ in the form of triangular waves with a period of $300 \mathrm{~km}$, and a smooth quasi-sinusoidal distribution for wind stress with typical amplitude of about $0.25 \mathrm{~N} \mathrm{~m}^{-2}$. Evolution of the ice thickness, shown in the fifth row of Fig. 2 (left), shows a configuration resulting in ice convergence with a discontinuity in the SIT distribution (row 4) between 1200 and $1300 \mathrm{~km}$. This discontinuity is visible in the SIV and SIT distributions of Figs. 3b and $3 \mathrm{c}$ (indicated by gray lines) and Fig. 4 (indicated by black lines), which show the true ice state at the start of day 4 as a reference. Throughout the text below, this feature of the true solution is identified as "ridging" behavior. Note that prescribed SIV and SIT boundary conditions are unbalanced with the wind forcing; the nonzero wind forcing near the boundaries results in unrealistic sharp decreases of SIT near those boundaries. Ideally, the ice thickness and concentration should decrease in these areas due to offshore ice transport under the influence of accelerating offshore winds. However, the SIC distribution is spatially invariant with $a(x)=1$ throughout each PIZ experiment and prevents this adjustment.

The standard errors for the stationary noise vector used to generate observations for wind stress, SIV, and 
TABLE 2. Experiments in the PIZ (OSSE-3, OSSE-3w, and OSSE-3n) assimilate noisy SIV and SIT observations while SIC is fixed at $a(x)=1$. Experiments in the MIZ (OSSE-4 and OSSE-4w) assimilate noisy SIV, SIC, and SIT observations while RPs are fixed at constant values. Noisy wind stress observations are assimilated in all cases subject to inexact wind forcing, and all experiments optimize the initial ice state.

\begin{tabular}{|c|c|c|c|}
\hline OSSE- & Description & Goal & Control (c) \\
\hline 3 & $\begin{array}{l}\text { PIZ with } a(x)=1 \text {, spatially varied RPs, } \\
\text { exact } \tau_{a} \text { data, inaccurate first-guess } \\
\text { state/RPs, and reasonable errors in } \\
\text { SIV/SIT data }\end{array}$ & $\begin{array}{l}\text { Evaluate the impact of RP optimization } \\
\text { under accurate wind forcing and } \\
\text { reasonable first-guess state/RPs }\end{array}$ & {$\left[u_{0}(x), h_{0}(x), P^{*}(x), e(x)\right]$} \\
\hline $3 w$ & $\begin{array}{l}\text { PIZ with } a(x)=1 \text {, spatially varied RPs, } \\
\text { inaccurate first-guess state } / \mathrm{RPs} / \tau_{a} \text {, and } \\
\text { reasonable errors in SIV } / \mathrm{SIT} / \tau_{a} \text { data }\end{array}$ & $\begin{array}{l}\text { Evaluate the impact of } \mathrm{RP} / \tau_{a} \text { optimization } \\
\text { under inaccurate wind forcing and } \\
\text { reasonable first-guess state/RPs }\end{array}$ & {$\left[u_{0}(x), h_{0}(x), \tau_{a}(x), P^{*}(x), e(x)\right]$} \\
\hline $3 n$ & $\begin{array}{l}\text { PIZ with } a(x)=1 \text {, spatially varied RPs, } \\
\text { inaccurate first-guess state } / \mathrm{RPs} / \tau_{a} \text {, and } \\
\text { reasonable errors in SIV } / \mathrm{SIT} / \tau_{a} \text { data }\end{array}$ & $\begin{array}{l}\text { Evaluate the impact of } \tau_{a} \text { optimization } \\
\text { under inaccurate wind forcing } \\
\text { reasonable first-guess state/RPs }\end{array}$ & {$\left[u_{0}(x), h_{0}(x), \tau_{a}(x)\right]$} \\
\hline 4 & $\begin{array}{l}\text { MIZ with } 0.2<a(x)<0.8, \text { spatially } \\
\text { uniform RPs, inaccurate first-guess state } / \tau_{a} \\
\text { and reasonable errors in SIC/SIT } / \tau_{a} \text { data with } \\
\text { low-accuracy SIV data }\end{array}$ & $\begin{array}{l}\text { Evaluate the impact of } \tau_{a} \text { optimization } \\
\text { under inaccurate wind forcing and } \\
\text { low-accuracy SIV observations }\end{array}$ & {$\left[u_{0}(x), h_{0}(x), a_{0}(x), \tau_{a}(x)\right]$} \\
\hline $4 w$ & $\begin{array}{l}\text { MIZ with } 0.2<a(x)<0.8, \text { spatially } \\
\text { uniform RPs, inaccurate first-guess state } / \tau_{a} \text {, } \\
\text { and reasonable errors in SIC/SIT } / \tau_{a} \text { data with } \\
\text { high-accuracy SIV data }\end{array}$ & $\begin{array}{l}\text { Evaluate the impact of } \tau_{a} \text { optimization } \\
\text { under inaccurate wind forcing and } \\
\text { high-accuracy SIV observations }\end{array}$ & {$\left[u_{0}(x), h_{0}(x), a_{0}(x), \tau_{a}(x)\right]$} \\
\hline
\end{tabular}

SIT are $0.13 \mathrm{~N} \mathrm{~m}^{-2}, 0.03 \mathrm{~m} \mathrm{~s}^{-1}$, and $0.22 \mathrm{~m}$, respectively, with associated maximum differences of $0.3 \mathrm{~N} \mathrm{~m}^{-2}$, $0.10 \mathrm{~m} \mathrm{~s}^{-1}$, and $0.6 \mathrm{~m}$. These errors target the respective $0.125 \mathrm{~N} \mathrm{~m}^{-2}, 0.025 \mathrm{~m} \mathrm{~s}^{-1}$, and 0.25 -m uncertainties in the data sources discussed in section 2c. Figure 3 illustrates the typical differences between the true solution and synthetic observations for these fields; while the true state variables evolve in time, the added noise component does not. To initialize the assimilation system, the true initial conditions for SIV and SIT are contaminated in a similar way as the data-supplying model, but with a longer spatial decorrelation scale as described in section 3. A first-guess control composed of these initial conditions, conventional RPs $P^{*}(x)=27500 \mathrm{~N} \mathrm{~m}^{-2}$ and $e(x)=2$, and exact wind stress characterizes OSSE-3.

The errors of the OSSE-3 first-guess solution with respect to true fields are shown in Fig. 2 (center). The small $\left(\sim 0.02 \mathrm{~m} \mathrm{~s}^{-1}\right)$ mean RMSE for first-guess SIV is due to the exact wind stress forcing provided in the experiment. The corresponding RMSE for SIT is more
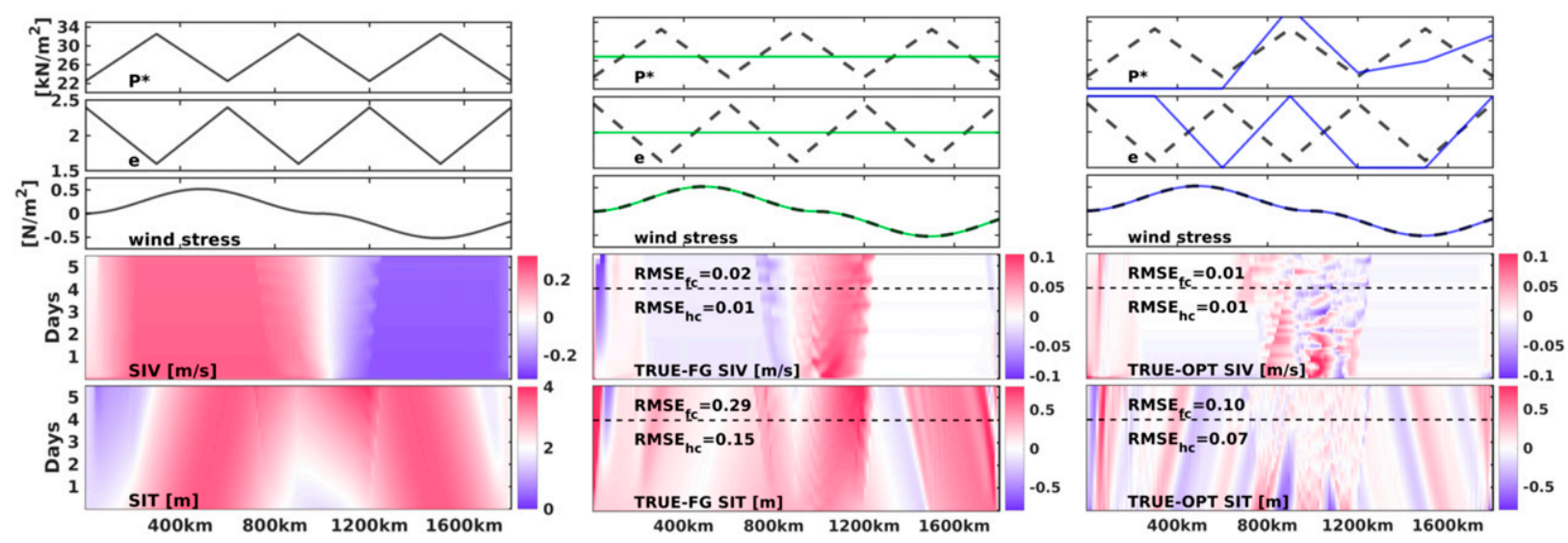

FIG. 2. (left) (top three rows) True parameter distributions and (bottom two rows) solutions for PIZ experiments (OSSE-3, OSSE-3w, and OSSE-3n). (top to bottom) Spatial variability of the stationary $P^{*}(x), e(x)$, and wind stress $\left[\tau_{a}(x)\right]$, and evolution of SIV and SIT, respectively. (center) OSSE-3 first-guess parameters and solution in relation to the true solution. (top three rows) True and first-guess distributions by the dashed black and solid green lines, respectively. (bottom two rows) Evolution of true minus first-guess errors for SIV and SIT, respectively. The horizontal dotted black line identifies the end of the assimilation window, with the RMSE for hindcast (hc) and forecast (fc) periods shown. (right) As in the center column, but for OSSE-3 optima. (top three rows) Blue lines identify the optimum parameters. 

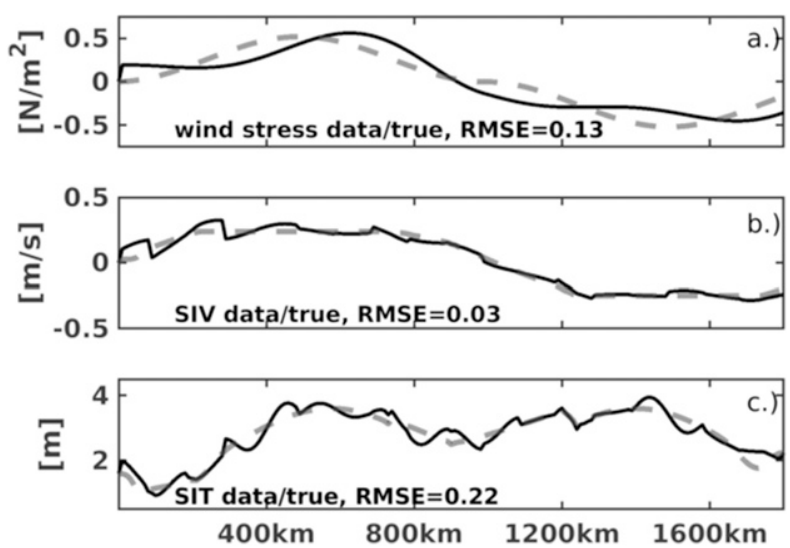

FIG. 3. Solid lines exemplify observations of the (a) wind stress, (b) SIV, and (b) SIT assimilated in PIZ OSSEs at the start of day 4 of the model run. Dashed lines represent the true solutions from which observational data are generated.

significant, equaling 0.19 and $0.29 \mathrm{~m}$ for the hindcast and forecast periods, respectively. Maximum differences in first-guess SIT occur in the domain between 900 and $1200 \mathrm{~km}$ and exceed $0.9 \mathrm{~m}$ in height, which is approximately $25 \%-30 \%$ of the local true SIT (Fig. 2, left). Figure 4 (left) underscores the significant qualitative difference between true and first-guess solutions. In particular, the first-guess thickness (identified by the green line) is rather smooth in the central part of the model domain (900-1300 km) and does not reflect the ridging discontinuities clearly seen in the true SIT (identified by the black line). There also exist problems near the boundaries; these are due to nonzero wind and fixed boundary conditions that are not in dynamical balance and excluded from the RMSE calculation.

The optimized $P^{*}(x)$ and $e(x)$ distributions and difference between optimized and true solutions are shown in Fig. 2 (right). Assimilation of SIV and SIT observations results in a spatial distribution of $P^{*}(x)$ more representative of the true solution, although this improvement is not uniform. The optimized distribution for $e(x)$ is generally inaccurate, which suggests that the elliptical yield curve eccentricity is of modest importance in the optimization. Specifically, one notes that the RMSE of the optimized RPs is roughly twice that of the constant, first-guess RPs in this case. The optimized solution yields better recovery of the $P^{*}(x)$ distribution with large reconstruction errors for $e(x)$ in the center of the domain, while the opposite [large errors for $P^{*}(x)$, better recovery for $e(x)$ ] appears to be true in the boundary regions. Further experiments suggest that the accuracy of the first-guess state and assimilated data strongly affect the accuracy of RP optimization. In particular, reduction of SIT errors in the first-guess initial control resulted in a more accurate recovery of $e(x)$. However, such results are natural in nonconvex optimization problems due to the presence of local minima.

The average effect of the SIT optimization is more profound for the forecast period where mean RMSE is $0.10 \mathrm{~m}$ ( $65 \%$ improvement over the first guess) and smaller for the hindcast period where mean RMSE is $0.07 \mathrm{~m}$ (53\% improvement over the first guess). Yet these average errors do not provide a complete picture; Fig. 4 (left) clearly demonstrates that the difference between first-guess and true SIT is $\sim 1 \mathrm{~m}$ near $1200 \mathrm{~km}$ and even larger near the boundaries, while optimized SIT is very close to the true SIT throughout most of modeled region after the first day.

Hindcast errors in the OSSE-3 optimal SIT are mostly due to the underestimation of SIT in the center of the domain around $900 \mathrm{~km}$ for the first day of simulation; the optimized SIV and SIT outperform the first-guess solution (e.g., Fig. 4, left) in the rest of the domain, boundary areas excluded. Additionally, the first-guess SIT errors are corrected by as much as $0.9 \mathrm{~m}$ over the central domain in the optimum. Also note that the optimal solution
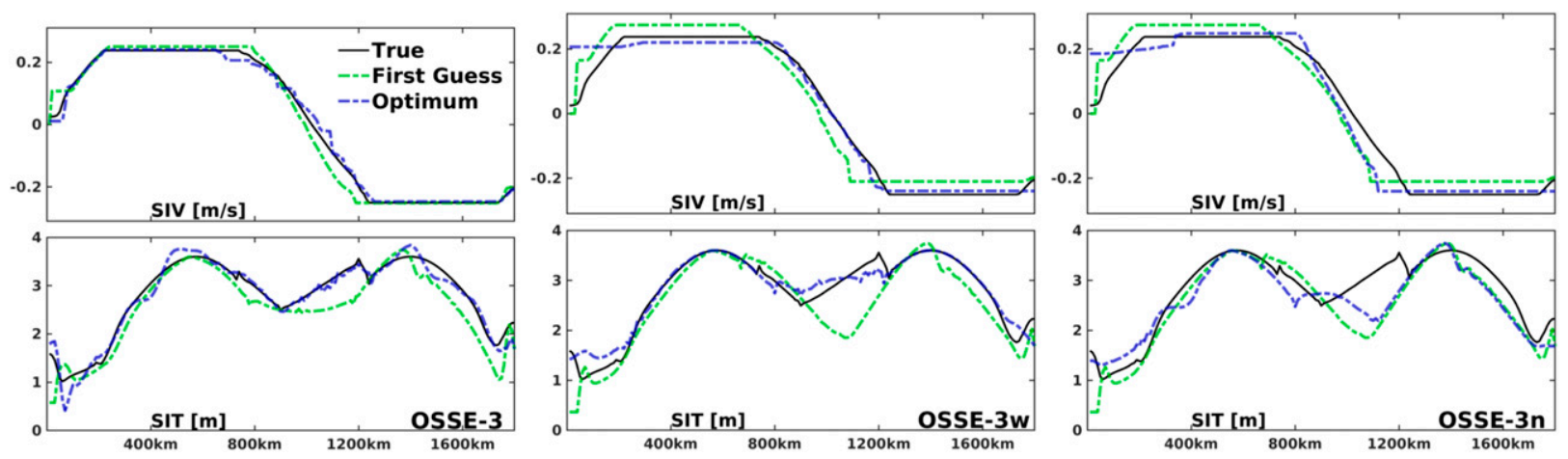

FIG. 4. (top) SIV and (bottom) SIT at the start of day 4 for each simulation. Solution distributions are shown for each of (left) OSSE-3, (center) OSSE-3w, and (right) OSSE-3n. True, first-guess, and optimal distributions are shown in solid black, dashed green, and dashed blue curves, respectively. The black curves are identical to the dashed gray curves in Fig. 3. 

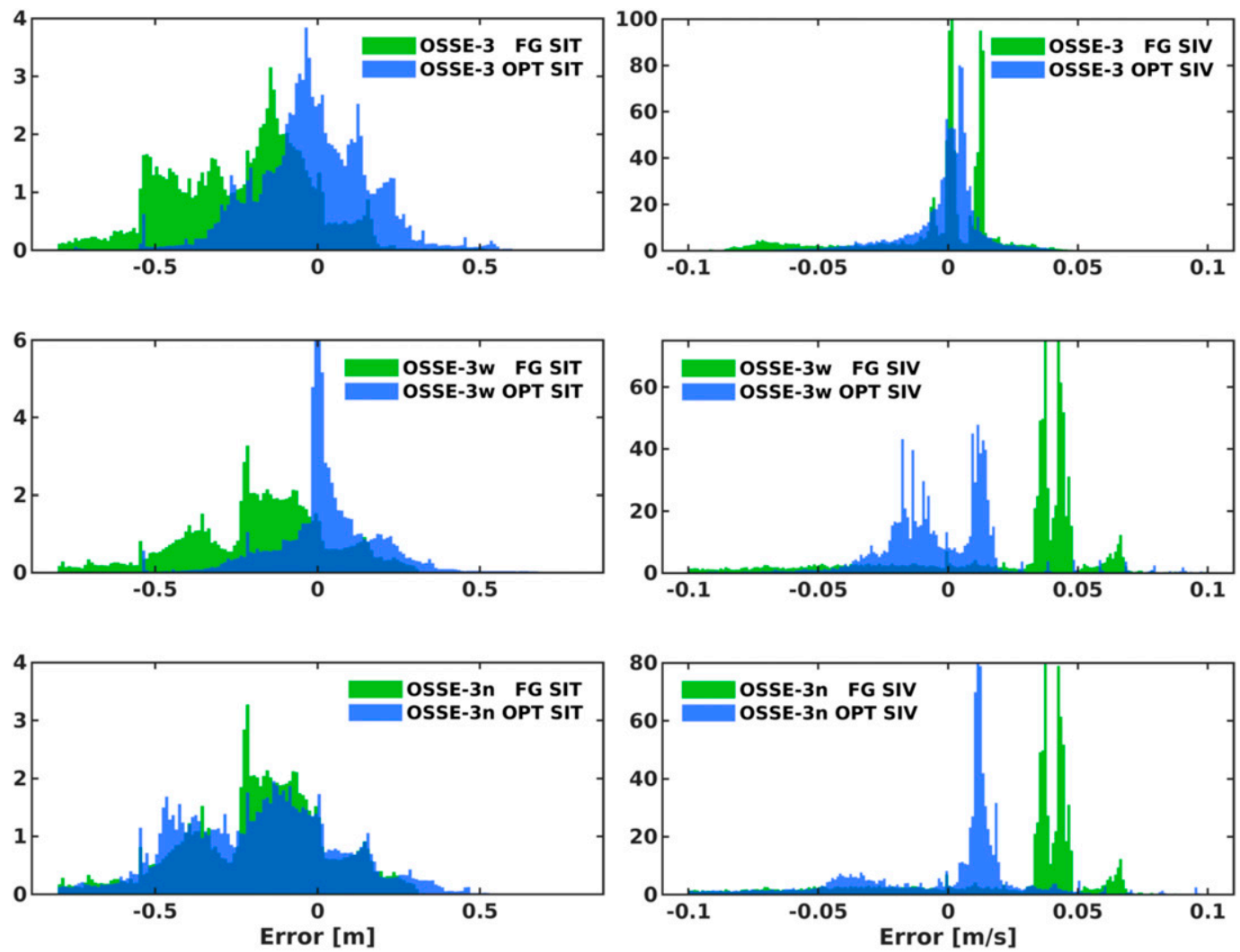

FIG. 5. Error distributions for (top) OSSE-3, (middle) OSSE-3w, and (bottom) OSSE-3n for (left) SIT and (right) SIV errors, calculated as experiment minus true, with first-guess solution errors presented in green and optimal solution errors in blue. Note that the first-guess errors of OSSE-3 differ from those of OSSE- 3 w and OSSE-3n due to being initialized with exact rather than noisy wind stress.

contains an SIT discontinuity between 1100 and $1300 \mathrm{~km}$ that is qualitatively the same as that found in the true solution, while the first-guess solution with uniform RPs does not present this feature.

The top row of Fig. 5 highlights the effect of RP variation and optimization on the error distributions in SIT and SIV. The first-guess solution of OSSE-3, where RP are constant, statistically underestimates SIT by about $0.3 \mathrm{~m}$, and the error structure is rather uniformly distributed over the range of $-0.5-0.0 \mathrm{~m}$. In contrast, the optimized SIT errors show statistically desirable qualities of low bias and approximate normality. Improvements in SIV error distribution are also evident.

The left and center columns of Fig. 6 presents the result of the more realistic OSSE-3w, which uses inaccurate wind stress in its first-guess control and observation data. The true-state, ice-state observations, and first-guess SIT/RPs are the same for this case as in OSSE-3 [see Figs. 2 (left) and 3 (left)], although the first-guess model trajectory differs as a result of the inaccurate wind forcing and, consequently, different initial SIV after spinup from rest. The control vector for OSSE-3w includes initial conditions, RPs, and wind stress. In the optimized solution, imperfect wind observations result in poor reconstruction of $P^{*}(x)$ and $e(x)$ in OSSE-3w (center column of Fig. 6, top panels), while the reconstructed wind stress is accurate. Despite this poor RP estimation, optimized SIV and SIT solutions (Fig. 6, bottom) are significantly closer to the true model state than the first-guess solution (left columns of Fig. 6, bottom). The applied assimilation system reduces SIV errors by $66 \%$ (from 0.03 to $0.01 \mathrm{~m} \mathrm{~s}^{-1}$ ) during reconstruction and by $50 \%$ (from 0.04 to $0.02 \mathrm{~m} \mathrm{~s}^{-1}$ ) during forecast, with averaged error reductions of $78 \%$ and $66 \%$ obtained for SIT hindcast and forecast, respectively. Similar to the OSSE-3 results, the major optimization impact is clearly observed in the central part of the modeled region away from 1000 to $1200 \mathrm{~km}$; in this subregion, the absolute differences between true and optimized SIV/SIT are about $0.01 \mathrm{~m} \mathrm{~s}^{-1}$ and $0.02 \mathrm{~m}$, respectively. Again, the structure of SIV and SIT error distributions (Fig. 5, middle row) shows approximate debiasing of both fields. However, the posterior errors for SIV retain the bimodal structure of the first-guess errors. 

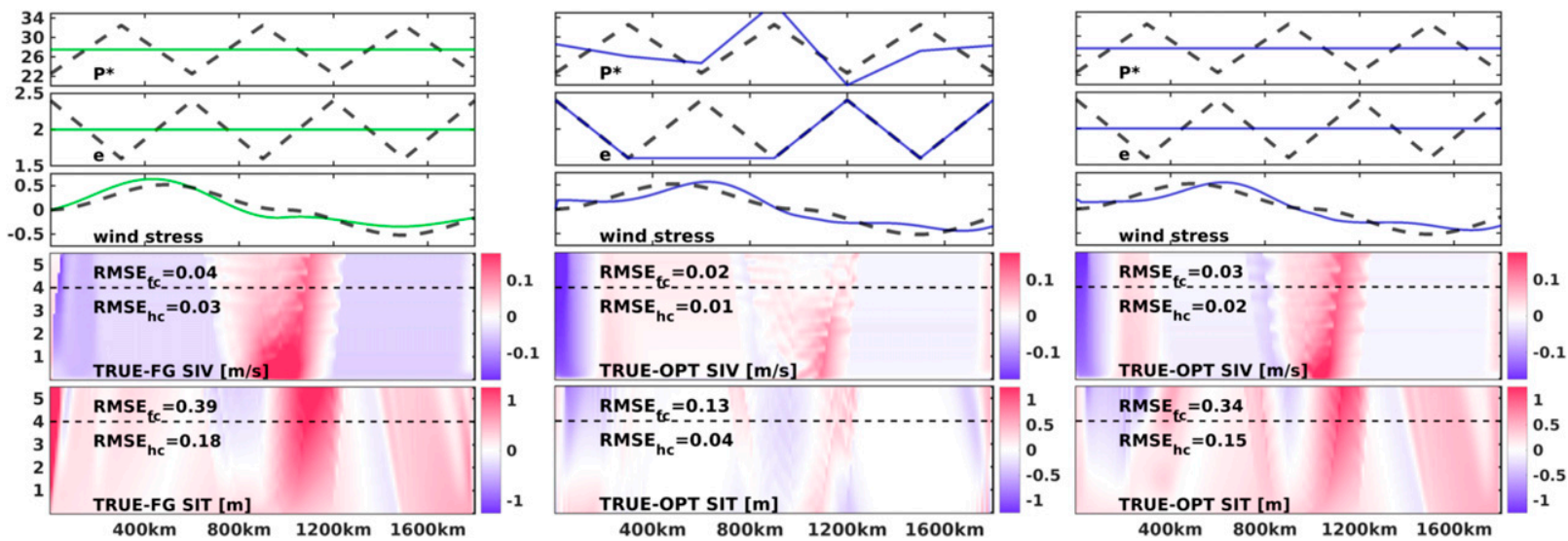

FIG. 6. (left) First-guess (top three rows) parameter distributions (green) and (bottom two rows) solution errors of OSSE-3w. (center) Relation of the OSSE-3w (top three rows) optimum distributions (blue) and (bottom two rows) solution errors. The layout mirrors those of the center and right columns of Fig. 2, respectively, showing results in relation to the true parameters/solution. (right) Optimized results of OSSE-3n in relation to the true (top three rows) parameters and (bottom two rows) solution.

The OSSE-3w instantaneous SIV and SIT optimal states at the end of the hindcast period (i.e., the start of day 4) are shown in Fig. 4 (center) (identified by the blue lines) and show significant superiority over the first-guess states (identified by the green lines). Interestingly, the inclusion of wind stress optimization provides a smoother SIT solution near the boundary than optimization of the initial state alone. In the ridging area (near $1200 \mathrm{~km}$ ), optimized SIT still demonstrates some level of discontinuity, but the feature is not as pronounced as in the OSSE-3 case with perfect wind. Small-scale noise in some optimal solutions is visible; see, for example, OSSE-3 optimal SIT around $1100 \mathrm{~km}$ in Fig. 4 (left). In supplemental OSSEs not detailed here, smoothness of SIV and SIT states was encouraged by including their squared second derivatives as additional cost function penalties; results suggest that such regularization is capable of eliminating this small-scale noise but inhibits formation of the desirable discontinuities in ridging regions. This problem may be formally overcome by implementing a more advanced nonuniform smoothing, but such an approach would require additional information to formulate a reasonable regularization constrain (e.g., Fomel 2007).

The right columns of Figs. 4 and 6 visualize OSSE-3n, which is similar to OSSE-3w but optimizes only initial conditions and wind stress while RP are held uniformly as in the first-guess solution. Figure 6 (right) shows that partial optimization still yields SIT distribution improvement, with optimized SIT hindcast and forecast improvements of $50 \%$ and $33 \%$, respectively. However, these improvements are modest compared to reductions of $78 \%$ and $66 \%$ achieved using full control in OSSE- $3 \mathrm{w}$. Figure 4 (right) clearly indicates that without variable RP included in the optimized control, there are marked differences from the true SIT in the central domain $(900-1300 \mathrm{~km})$ and the optimized SIT lacks the discontinuity near $1200 \mathrm{~km}$. The OSSE-3n optimal initial state and wind stress are otherwise similar to those of OSSE-3w, which confirms that variable RP significantly affect the evolution of the sea ice state. This is further supported by examination of the optimized SIT error structure for OSSE-3n (Fig. 5, bottom left), which shows little change from the corresponding first-guess errors and continues to underestimate SIT.

Optimization of the initial conditions in OSSE-3n has a minor impact on the forecast SIV, which remains close to the first-guess SIV for the entire forecast period (see right columns of Figs. 4 and 6). This similarity likely results from identical rheological parameters and a small difference between first-guess wind stress and wind stress observations. The optimization does, however, reduce SIV bias from $\sim 0.04$ to $\sim 0.01 \mathrm{~m} \mathrm{~s}^{-1}$ (Fig. 5, bottom right). Thus, the comparison of OSSE- $3 \mathrm{w}$ and OSSE-3n optima clearly indicates the significant impact that $P^{*}(x)$ and $e(x)$ optimization has on obtaining accurate short-range sea ice forecasts in the idealized PIZ region.

To more clearly distinguish the impact of $P^{*}(x)$ and $e(x)$ variability, two additional OSSEs were performed where the optimized solutions were obtained with either $P^{*}$ or $e$ taken as uniform. Results showed that optimization over each nonuniform RP independently provides approximately the same hindcast/forecast mean errors in SIV and SIT distributions, and were larger than errors in OSSE-3w. However, optimization of $e(x)$ with constant $P^{*}(x)$ did not provide reconstruction of the SIT discontinuities discussed above. This result suggests that the 1D $e(x)$ does not influence the formation of discontinuities. 

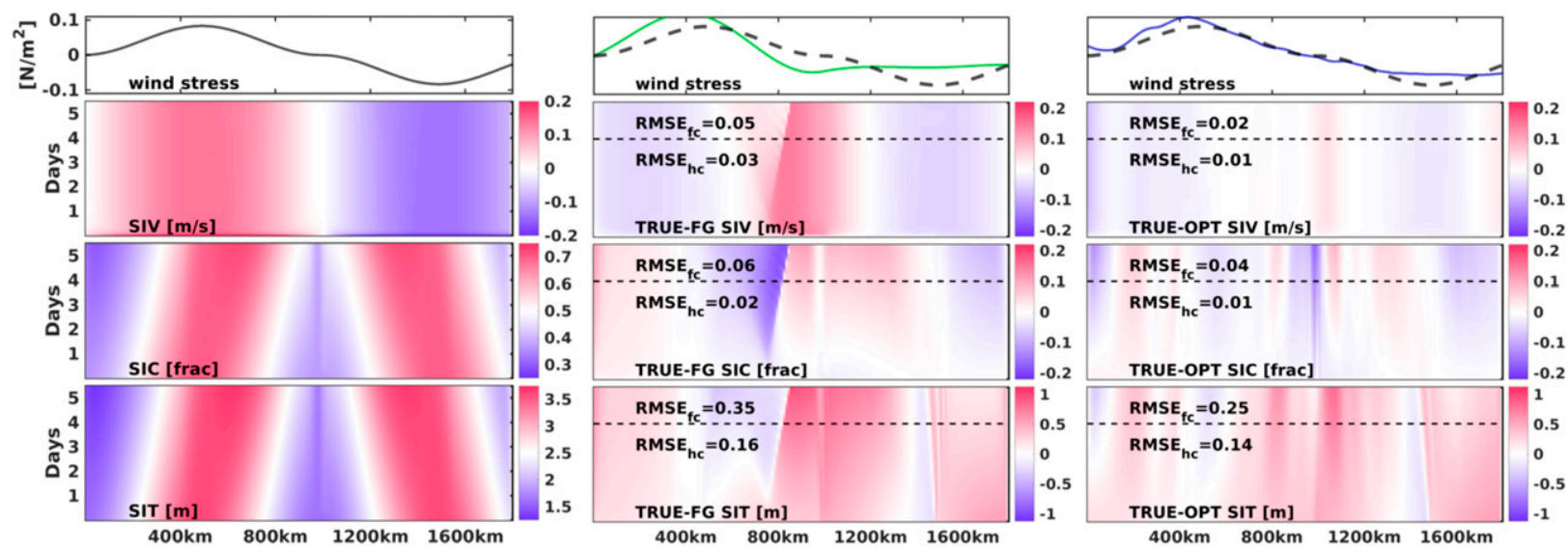

FIG. 7. (left) (top) True wind stress and (bottom three rows) solution for MIZ experiments (OSSE-4, OSSE-4w). (top to bottom) Spatial variability of the wind stress $\tau_{a}$ and evolution of sea ice state. (center) OSSE-4 first-guess parameters and solution in relation to the true solution. (top) True and first-guess $\tau_{a}(x)$ is shown by the dashed gray and green lines, respectively. (bottom three rows) Evolution of true minus first-guess errors for SIV, SIC, and SIT, respectively. The dotted black line identifies the end of the assimilation window, with the RMSE for hindcast (hc) and forecast (fc) periods shown. (right) As in the center column, but for OSSE-4 optima, with blue lines showing the optimal parameters. Rheological parameters $P^{*}(x)=27500 \mathrm{~N} \mathrm{~m}^{-2}$ and $e(x)=2$ remain fixed throughout and are not shown. Black/gray lines in the top panels illustrate the true parameter distribution.

In all cases, identified ridges are located in true and optimized solutions where $P^{*}(x)$ attains a local minimum. Therefore, optimization with respect to spatially variable $P^{*}(x)$ appears to be more important for shortrange hindcast/forecast than $e(x)$ in the $1 \mathrm{D}$ case; this is also suggested by the quality of SIT/SIV reconstruction in OSSE-3 despite inaccurate reconstruction of $e(x)$.

OSSE-3 shows that optimized RPs have larger RMSE than the constant first-guess RPs, but this is not the case for OSSE-3w. The optimized $P^{*}(x)$ in both cases, however, is most accurate where SIV is convergent and the VP rheology has a larger impact on model dynamics. Where ice convergence is small or the ice diverges, there is limited constraint within the VarDAS to determine these RPs as their dynamical role is greatly reduced. This suggests that a priori knowledge of SIV convergence during the hindcast period could be used in practice to reduce the control vector dimension by controlling RPs only in those regions; this would further aid convergence of the optimization by deemphasizing poorly constrained control elements.

An interesting result of OSSE- 3 and OSSE- $3 w$ together is that the first-guess solutions with constant RPs show a clear negative SIT bias with respect to the true SIT solution, while optimization using spatially variable $P^{*}(x)$ and $e(x)$ significantly reduces this underestimation (cf. Fig. 5, left panels). This is in strong agreement with aforementioned results of Juricke et al. (2013), which found that simulations using varied $P^{*}$ led to central Arctic SIT distributions that could not be reproduced with fixed $P^{*}$. Observed biases in sea ice thickness and volume in some modeling systems (e.g., the Arctic Cap
Nowcast/Forecast System; Posey et al. 2015) may be reduced after conditioning SIT initialization on observations (Allard et al. 2018), and the results of the OSSEs here suggest that observational conditioning with variable RPs may provide similar benefits in more realistic applications.

\section{b. Marginal ice zone}

The MIZ regime greatly limits the role rheological stresses play in the sea ice dynamical balance; the VP internal pressure with $80 \%$ SIC is less than $2 \%$ of its value at $100 \%$ SIC with other parameters unchanged. One expects the impact of spatial variability in RP to be negligible as well, so spatially uniform parameters, $P^{*}=27500 \mathrm{~N} \mathrm{~m}^{-2}$ and $e=2$, reflect these assumptions in MIZ OSSEs presented. To avoid instabilities in model simulation, the amplitude of the supplied wind stress $\tau_{a}(x)$ is reduced to avoid violation of CFL conditions. This reflects an assumption that $\tau_{a}(x)$ now includes the increased magnitude of ocean drag associated with lower SIC (Steele et al. 1989). The observational wind stress error amplitude is likewise reduced to $0.04 \mathrm{~N} \mathrm{~m}^{-2}$ to maintain a proportional signal-to-noise ratio.

The MIZ OSSEs further assume that true initial conditions for SIC are proportional to true SIT for convenience. The evolution of the true MIZ solution is shown in Fig. 7a. Ice in the model solution moves toward to the center of the domain similarly to the PIZ case, but lacks the marked discontinuities in the ice-state variables that were present in the true PIZ solution.

The generation of observations and first-guess solution for MIZ OSSEs follows those described previously 
for OSSE-3w. Figure 7 (center) shows the evolution of the first-guess solution, and Fig. 8 provides an example of observations at the start of day 4 that now include SIC data. First-guess and observed wind stress differ significantly from the true wind stress with mean RMSEs of 0.04 and $0.02 \mathrm{~N} \mathrm{~m}^{-2}$, respectively, and are aligned with $10-\mathrm{m}$ wind error estimates calculated from reanalysis products (Jakobson et al. 2012). In OSSE-4, the optimization was conducted with respect to initial-state conditions (which now includes SIC) and then wind stress, and results of the optimization are shown in Fig. 7 (right).

The optimal solution of OSSE-4 is clearly an improvement over the first-guess solution, with relative RMSE reductions for hindcast (forecast) SIV, SIC, and SIT distributions of $67 \%(60 \%), 50 \%(33 \%)$, and $13 \%$ (29\%), respectively. In particular, Fig. 9a compares true, first-guess, and optimized solutions at the start of day 4 and suggests that the most significant improvements occur in optimized SIV, which is 5 times closer to the true solution than the first-guess SIV. Interestingly, the optimized $\tau_{a}(x)$ is much closer to the true wind stress with RMSE of $\sim 0.01 \mathrm{~N} \mathrm{~m}^{-2}$ despite large errors $\left(\sim 0.04 \mathrm{~N} \mathrm{~m}^{-2}\right)$ in the supplied wind stress observations. This is a consequence of assimilating SIV observations, which relate directly to wind velocity; in the absence of the internal rheological stress and ocean drag, sea ice velocities are linearly related to the atmospheric wind stress by Eq. (1). This suggests prerequisite use of SIV observations for estimation of atmospheric stress in the MIZ. Further, it suggests SIV may be utilized in conjunction with other ice-state estimates as additional atmospheric observations to improve atmospheric reconstruction and forecast.

To better illustrate the possibility of refining wind stress on the basis of SIV observations, OSSE- $4 \mathrm{w}$ is conducted identically to OSSE-4 only with more accurate SIV observations; the data are contaminated with noise of $1 / 3$ the amplitude $\left(\sim 0.01 \mathrm{~m} \mathrm{~s}^{-1}\right)$ of those used in OSSE- 4 as shown in Fig. 8 b (thin black line). The optimized solution is shown in Fig. 10 and improves the reconstructed wind stress as well as ice-state distributions beyond the results of OSSE-4. With all else equal, the threefold increase in accuracy of assimilated SIV observations yields a threefold decrease in reconstructed wind stress errors (viz., from $\sim 0.009 \mathrm{~N} \mathrm{~m}^{-2}$ in OSSE-4 to $\sim 0.003 \mathrm{~N} \mathrm{~m}^{-2}$ in OSSE-4w). This result is based on the form of $\tau_{a}$ that incorporates the water drag of a zerovelocity ocean; estimates of ocean velocity are needed to extend this result to realistic cases where the water drag depends on ocean velocity.

The comparison of OSSE-4 and OSSE-4w suggests that high-accuracy SIV observations may significantly benefit hindcast/forecast quality of the sea ice state and of weather forecast systems as well. Such accurate SIV
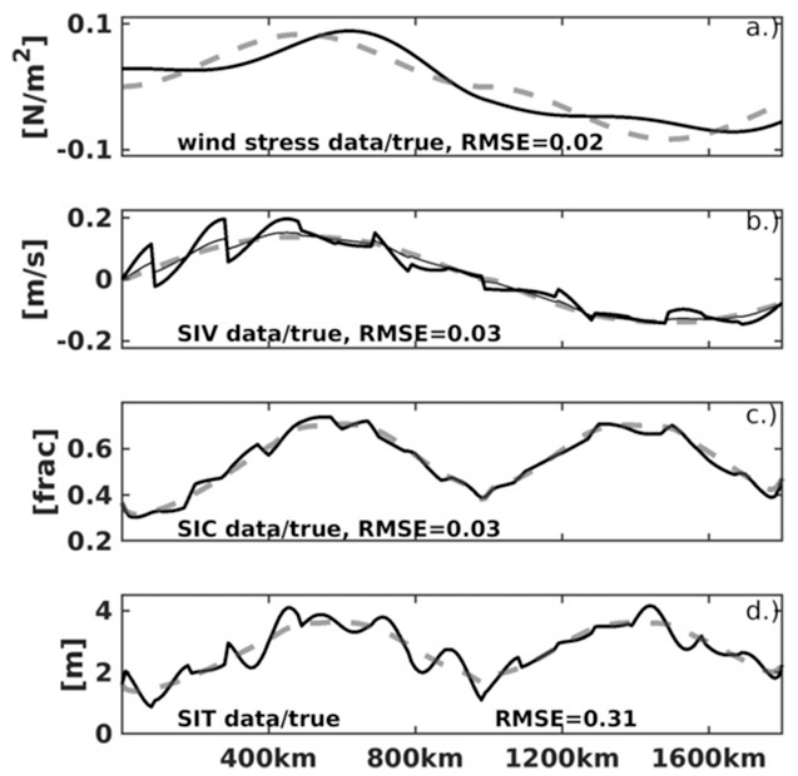

FIG. 8. Observations of (a) wind stress, (b) SIV, (c) SIC, and (d) SIT used as model constraints at the start of day 4. Thick solid lines show example observations for OSSE-4, while the dashed lines track the corresponding true solution of the model run. The RMSEs identify the statistical difference between true state and synthetic data throughout the assimilation window. The thin black line of the SIV plot represents the high-accuracy $\left(0.01 \mathrm{~m} \mathrm{~s}^{-1}\right.$ RMSE) ice velocity observations used in OSSE-4w.

observations are typically obtained from analysis using the maximum cross-correlation (MCC) method applied to sequential SAR images (Fily and Rothrock 1987; Komarov and Barber 2014). In this method, the presence of the open-water generally allows for more accurate estimation of the cross correlations between two data images. Based on this, one expects more accurate SIV estimates in the MIZ than in the PIZ where ice concentration is more homogeneous.

\section{Summary}

The presented study addresses the feasibility of optimizing spatially varied rheological parameters (RPs) and wind stress $\tau_{a}$ by assimilating simulated satellite observations of sea ice velocity, concentration, and thickness (SIV, SIC, and SIT, respectively) with realistic errors. The approach to this problem used a variational data assimilation system (VarDAS) constructed from a simple nonlinear $1 \mathrm{D}$ sea ice model previously used to analyze a modified rheology and numerical scheme (König Beatty and Holland 2010; Auclair et al. 2017) together with its associated adjoint model. Based on differing dynamical regimes, observation synthesis simulation experiments (OSSEs) were organized separately for the pack ice zone (PIZ; where SIC is maintained at 

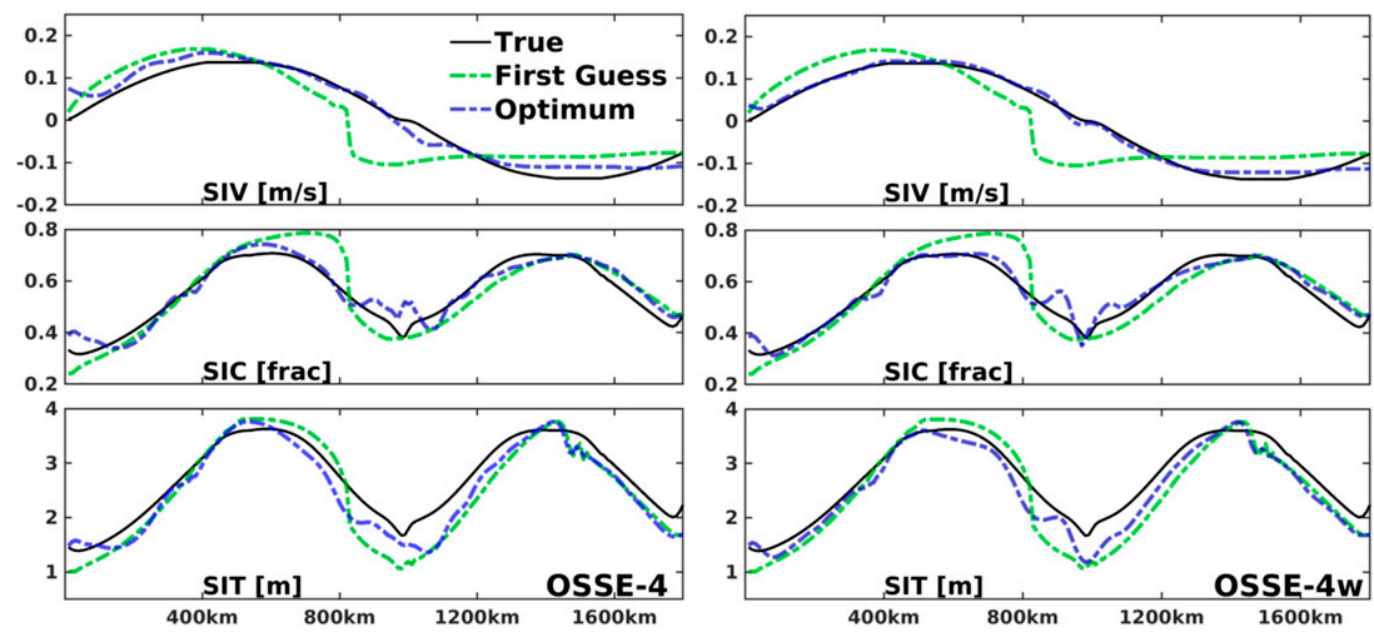

FIG. 9. (left) OSSE-4 and (right) OSSE-4w model states at the start of day 4 are shown for (top) SIV, (middle) SIC, and (bottom) SIT distributions.

$100 \%$ ) and the marginal ice zone (MIZ; where SIC is between $20 \%$ and $80 \%$ ).

OSSEs in this study simulated realistic high-accuracy SIV and SIC observations with $0.025 \mathrm{~m} \mathrm{~s}^{-1}$ and $5 \%$ RMSEs, respectively, and assimilated representative observations with $0.03 \mathrm{~m} \mathrm{~s}^{-1}$ and $3 \%$ RMSEs, respectively, after enforcing spatial regularity. Observations of SIC are not used in the PIZ experiments. In a similar way, moderately accurate wind stress and SIT observations were simulated with uncertainties of $0.125 \mathrm{~N} \mathrm{~m}^{-2}$ $\left(0.04 \mathrm{~N} \mathrm{~m}^{-2}\right)$ and $0.25 \mathrm{~m}(0.35 \mathrm{~m})$, respectively, in the PIZ (MIZ). After imposing realistic decorrelation scale lengths on these errors, PIZ (MIZ) experiments ingested wind stress and SIT observations with $0.13 \mathrm{~N} \mathrm{~m}^{-2}$ $\left(0.04 \mathrm{~N} \mathrm{~m}^{-2}\right)$ and $0.22 \mathrm{~m}(0.31 \mathrm{~m})$ RMSEs, respectively. Actual SIT observations are less accurate than assumed in these experiments, although forthcoming observational platforms and methodologies should provide SIT observations with uncertainties less than those currently available and comparable to those simulated.

In OSSEs for the PIZ, optimization of variable rheological parameters resulted in improved hindcast/forecast of the sea ice-state variables. Of note is that this improvement is achieved even when the RMSE of optimum RPs is larger than those of the first guess when the true solution has spatially variable parameters (as in OSSE-3). The flexible reconstruction of RPs where ice moves uniformly suggests that variable RPs may be of reduced importance in those regions, and prior knowledge of divergence-free areas could be used to deemphasize RP control where it has little practical benefit. The PIZ OSSEs showed that optimized variable RPs yielded a model evolution that corrected SIT underestimation of the first-guess solutions, unlike experiments using traditional
RPs. This results agrees with those of Juricke et al. (2013), which found simulations using a stochastically perturbed strength parameter cannot be reproduced with a fixed, uniform $P^{*}$. OSSE results also show that partial optimization using a subset of rheological terms may significantly improve hindcast/forecast of the sea ice state.

Individual optimizations of $P^{*}(x)$ and $e(x)$ produced similar RMSE improvements in SIV and SIT states in the case of full ice cover. However, optimization of

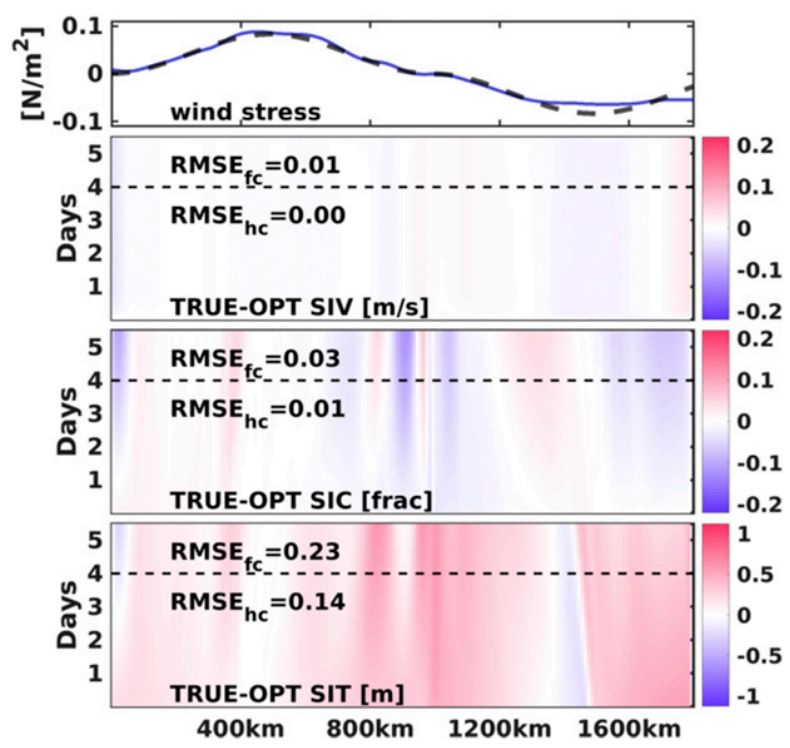

FIG. 10. Errors for the optimized solution for the OSSE-4w (highaccuracy ice velocity observations) are shown as in Fig. 7 (right). (top to bottom) Optimized wind stress distribution, and evolution of errors in the optimal SIV, SIC, and SIT, respectively. Standard errors of the optimized solution with respect to the true solution are shown for each forecast and hindcast region. 
strength parameter $P^{*}(x)$ allowed solutions to form discontinuous SIT distributions that can be associated with ice-ridging regions. Results also show that accurate state reconstructions are achieved when the optimized $e(x)$ is inaccurate, suggesting that its role is of less relative importance in the 1D case than wind stress and spatially variable $P^{*}(x)$. However, sea ice-state reconstruction strongly depends also on accuracy of the wind stress forcing and assimilated observations.

In the MIZ, the effects of internal sea ice stress are negligible and optimization of the rheological parameters are relatively unimportant. Instead, the conducted OSSEs revealed that wind stress is successfully optimized through the assimilation of SIV observations in the idealized case where water drag is independent of ocean velocity. This results mostly from the known linear relationship between wind stress and sea ice velocity in the MIZ, and consequently the quality of the wind stress correction is roughly proportional to the accuracy of assimilated SIV observations. In realistic coupled sea ice-ocean model applications, it is necessary to account for ocean friction in order to accurately estimate the wind stress. This may be achievable through the assimilation of additional ocean-side observations such as upper-ocean velocities and sea surface height.

As pack ice is the prevalent regime over most of the central Arctic Ocean throughout the year, these results clearly indicate practical benefits of including spatially varied $P^{*}(x)$ and $e(x)$ into sea ice models and motivate the development of proper optimization algorithms to handle the more dynamically complex 2D case. Notably, optimizations using variable RPs resulted in solutions with considerably reduced negative SIT bias than those using fixed RPs. This may partially explain the source of observed SIT underestimation in some sea ice models employing a set of constant RPs. However, thermodynamic components of modern sea ice models, which are not considered in the presented short-term experiments, need to be considered in assessing impacts and biases in longer simulations.

Continued development of this algorithm will also allow for estimation of local wind stress in the MIZ, which composes a significant portion of the Arctic Ocean in the summer months. At present, the simplified model is limited to one dimension, and a proper MIZ implementation must account for more complicated physical considerations such as imposed wind stress curl, Coriolis coupling of velocities, effects of ice-ocean drag, sea surface tilt, and different ice types that also affect the accuracy of assimilated observations. More research and development are needed to extend the idealized experimental results to practical settings.
The sea ice model is nonlinear, and the cost function of the VarDAS has multiple local minima; the calculated optimal solution therefore depends on the firstguess solution. However, assimilation of a significant volume of sea ice observations should provide sufficient correction and constraint to the model evolution that the optimization scheme targets an optimum that is "close enough" to the "true" solution after several assimilation cycles. This suggests that future developments toward realistic applications implementing the assimilation methodology described here should focus on hindcast periods with abundant data.

Also, the modern analysis of SAR images and other satellite observations permits accurate identification of different ice types (e.g., multiyear/first-year ice, deformed first-year ice) (Zakhvatkina et al. 2013, 2017) and leads (Murashkin et al. 2018). New methods of processing satellite data resolve additional sea ice characteristics that affect its material properties, such as ice age (Korosov et al. 2018), which has long been known to affect ice strength (Weeks and Assur 1967; Timco and Weeks 2010). Such information may be efficiently used to reduce of the dimension of the rheological control vector through regional definitions. For example, one could partition a pan-Arctic model domain into subregions based on the maturity and type of sea ice present, and prescribe to each a set of local strength distribution $P^{*}(x)$. Such an approach, of course, requires experimentation to empirically determine optimal RPs for each ice category. Furthermore, the ongoing work of resolving sea ice properties from remote sensing provides a base of information necessary to establish a proper regularization scheme (e.g., through spatially variable smoothing) for the optimization problem presented here. The need for sophisticated regularization is clear in some pack ice OSSEs; without it, large errors in observational data may produce artificially noisy ice states, while imposing spatially homogeneous regularization prevents the formation of physically relevant and appropriate discontinuities.

These results are encouraging, although they are based on a relatively simple $1 \mathrm{D}$ sea ice model employing the VP rheology proposed by Hibler (1979). The most widely used sea ice models are two-dimensional and implement the elastic-VP scheme, which is more efficient for parallel implementation on supercomputers (Hunke et al. 2010; Bouillon et al. 2013). The more complicated numerical formulation of such models may result in more-difficultto-avoid instabilities in their associated adjoint models. Development of full tangent linear and adjoint models for existing community sea ice models (e.g., CICE) is a longterm project. In the meantime, continued research and OSSEs with a more realistic $2 \mathrm{D}$ sea ice model are the 
next steps toward achieving locally optimal rheology through data assimilation.

Acknowledgments. The authors are indebted to J. Hutchings and two anonymous reviewers for valuable discussion and recommendations during revision of this article. J.N. Stroh received no funding or support during this research, and thanks the University of Nevada Reno for liberal access to library resources during document preparation. The NRL authors were supported by the Office of Naval Research Program Elements 0603207N (Navy Earth System Prediction Capability) and 0602435N (Arctic Data Assimilation). Oceana Francis was supported by the Coastal Hydraulics Engineering Resilience (CHER) Lab, the Civil and Environmental Engineering Department, and the Sea Grant College Program at the University of Hawai'i at Mānoa.

\section{REFERENCES}

Alexandrov, V., S. Sandven, J. Wahlin, and O. Johannessen, 2010: The relation between sea ice thickness and freeboard in the Arctic. Cryosphere, 4, 373-380, https://doi.org/10.5194/tc-4-373-2010.

Allard, R. A., and Coauthors, 2018: Utilizing CryoSat-2 sea ice thickness to initialize a coupled ice-ocean modeling system. Adv. Space Res., 62, 1265-1280, https://doi.org/10.1016/ j.asr.2017.12.030.

Auclair, J.-P., J.-F. Lemieux, L. B. Tremblay, and H. Ritchie, 2017: Implementation of Newton's method with an analytical Jacobian to solve the 1D sea ice momentum equation. J. Comput. Phys., 340, 69-84, https://doi.org/10.1016/j.jcp.2017.02.065.

Bouillon, S., and P. Rampal, 2015a: On producing sea ice deformation data sets from SAR-derived sea ice motion. Cryosphere, 9, 663-673, https://doi.org/10.5194/tc-9-663-2015.

$\ldots$, and —_, 2015b: Presentation of the dynamical core of neXtSIM, a new sea ice model. Ocean Modell., 91, 23-37, https://doi.org/10.1016/j.ocemod.2015.04.005.

_- T. Fichefet, V. Legat, and G. Madec, 2013: The elasticviscous-plastic method revisited. Ocean Modell., 71, 2-12, https://doi.org/10.1016/j.ocemod.2013.05.013.

Cullather, R. I., and M. G. Bosilovich, 2011: The moisture budget of the polar atmosphere in MERRA. J. Climate, 24, 28612879, https://doi.org/10.1175/2010JCLI4090.1.

Cummings, J. A., and O. M. Smedstad, 2013: Variational data assimilation for the global ocean. Data Assimilation for Atmospheric, Oceanic and Hydrologic Applications, Vol. II, Springer, 303-343.

Danilov, S., Q. Wang, R. Timmermann, N. Iakovlev, D. Sidorenko, M. Kimmritz, T. Jung, and J. Schröter, 2015: Finite-element sea ice model (FESIM), version 2. Geosci. Model Dev., 8, 1747-1761, https://doi.org/10.5194/gmd-8-1747-2015.

Dee, D. P., and Coauthors, 2011: The ERA-Interim reanalysis: Configuration and performance of the data assimilation system. Quart. J. Roy. Meteor. Soc., 137, 553-597, https://doi.org/10.1002/qj.828.

Dukowicz, J. K., 1997: Comments on "Stability of the viscous-plastic sea ice rheology." J. Phys. Oceanogr., 27, 480-481, https://oi.org/ 10.1175/1520-0485(1997)027<0480:COSOTV>2.0.CO;2.

Fenty, I., D. Menemenlis, and H. Zhang, 2017: Global coupled sea ice-ocean state estimation. Climate Dyn., 49, 931-956, https:// doi.org/10.1007/s00382-015-2796-6.
Fily, M., and D. Rothrock, 1987: Sea ice tracking by nested correlations. IEEE Trans. Geosci. Remote Sens., GE-25, 570-580, https://doi.org/10.1109/TGRS.1987.289836.

Fomel, S., 2007: Shaping regularization in geophysical-estimation problems. Geophysics, 72, R29-R36, https://doi.org/10.1190/ 1.2433716.

Forget, G., J.-M. Campin, P. Heimbach, C. Hill, R. Ponte, and C. Wunsch, 2015: ECCO version 4: An integrated framework for non-linear inverse modeling and global ocean state estimation. Geosci. Model Dev., 8, 3071-3104, https://doi.org/ 10.5194/gmd-8-3071-2015.

Goodier, J. N., and P. G. Hodge, 1958: Elasticity and Plasticity: The Mathematical Theory of Elasticity. Vol. 1. John Wiley and Sons, $152 \mathrm{pp}$.

Gray, J., and P. D. Killworth, 1995: Stability of the viscousplastic sea ice rheology. J. Phys. Oceanogr., 25, 971-978, https://doi.org/10.1175/1520-0485(1995)025<0971:SOTVPS> 2.0.CO;2.

Harder, M., and H. Fischer, 1999: Sea ice dynamics in the Weddell Sea simulated with an optimized model. J. Geophys. Res., 104, 11151-11162, https://doi.org/10.1029/1999JC900047.

Heimbach, P., 2008: The MITgcm/ECCO adjoint modelling infrastructure. CLIVAR Exchanges, No. 44, International CLIVAR Project Office, Southampton, United Kingdom, 13-17.

Hibler, W. D., III, 1977: A viscous sea ice law as a stochastic average of plasticity. J. Geophys. Res., 82, 3932-3938, https:// doi.org/10.1029/JC082i027p03932.

- 1979: A dynamic thermodynamic sea ice model. J. Phys. Oceanogr., 9, 815-846, https://doi.org/10.1175/1520-0485(1979) $009<0815$ :ADTSIM > 2.0.CO;2.

— nual fluctuations of Arctic sea ice. J. Phys. Oceanogr., 12, 1514-1523, https://doi.org/10.1175/1520-0485(1982)012<1514: OMSAIF $>2.0 . \mathrm{CO} ; 2$.

Houtekamer, P. L., and H. L. Mitchell, 1998: Data assimilation using an ensemble Kalman filter technique. Mon. Wea. Rev., 126, 796-811, https://doi.org/10.1175/1520-0493(1998)126<0796: DAUAEK $>2.0 . \mathrm{CO} ; 2$.

Hunke, E. C., and J. Dukowicz, 1997: An elastic-viscous-plastic model for sea ice dynamics. J. Phys. Oceanogr., 27, 1849-1867, https:// doi.org/10.1175/1520-0485(1997)027<1849:AEVPMF>2.0.CO;2.

- W. H. Lipscomb, A. K. Turner, N. Jeffery, and S. Elliott, 2010: CICE: The Los Alamos Sea Ice Model documentation and software user's manual version 4.1. Los Alamos National Laboratory Rep. LA-CC-06-012, 76 pp.

Jakobson, E., T. Vihma, T. Palo, L. Jakobson, H. Keernik, and J. Jaagus, 2012: Validation of atmospheric reanalyses over the central Arctic Ocean. Geophys. Res. Lett., 39, L10802, https:// doi.org/10.1029/2012GL051591.

Juricke, S., P. Lemke, R. Timmermann, and T. Rackow, 2013: Effects of stochastic ice strength perturbation on Arctic finite element sea ice modeling. J. Climate, 26, 3785-3802, https:// doi.org/10.1175/JCLI-D-12-00388.1.

Komarov, A. S., and D. G. Barber, 2014: Sea ice motion tracking from sequential dual-polarization RadarSat-2 images. IEEE Trans. Geosci. Remote Sens., 52, 121-136, https://doi.org/ 10.1109/TGRS.2012.2236845.

König Beatty, C., and D. M. Holland, 2010: Modeling landfast sea ice by adding tensile strength. J. Phys. Oceanogr., 40, 185-198, https://doi.org/10.1175/2009JPO4105.1.

Korosov, A. A., and Coauthors, 2018: A new tracking algorithm for sea ice age distribution estimation. Cryosphere, 12, 2073-2085, https://doi.org/10.5194/tc-12-2073-2018. 
Kreyscher, M., M. Harder, and P. Lemke, 1997: First results of the Sea-Ice Model Intercomparison Project (SIMIP). Ann. Glaciol., 25, 8-11, https://doi.org/10.3189/S0260305500013719.

,,--- , and G. M. Flato, 2000: Results of the Sea Ice Model Intercomparison Project: Evaluation of sea ice rheology schemes for use in climate simulations. J. Geophys. Res., 105, 11 299-11 320, https://doi.org/10.1029/1999JC000016.

Laxon, S. W., and Coauthors, 2013: CryoSat-2 estimates of Arctic sea ice thickness and volume. Geophys. Res. Lett., 40, 732-737, https://doi.org/10.1002/grl.50193.

Le Dimet, F.-X., and O. Talagrand, 1986: Variational algorithms for analysis and assimilation of meteorological observations: Theoretical aspects. Tellus, 38A, 97-110, https://doi.org/ 10.3402/tellusa.v38i2.11706.

Lemieux, J.-F., B. Tremblay, S. Thomas, J. Sedláček, and L. A Mysak, 2008: Using the preconditioned generalized minimum residual (GMRES) method to solve the sea-ice momentum equation. J. Geophys. Res., 113, C10004, https://doi.org/10.1029/ 2007JC004680.

- F. Dupont, P. Blain, F. Roy, G. C. Smith, and G. M. Flato, 2016: Improving the simulation of landfast ice by combining tensile strength and a parameterization for grounded ridges. J. Geophys. Res. Oceans, 121, 7354-7368, https://doi.org/10.1002/ 2016JC012006.

Lindsay, R., and J. Zhang, 2006: Assimilation of ice concentration in an ice-ocean model. J. Atmos. Oceanic Technol., 23, 742 749, https://doi.org/10.1175/JTECH1871.1.

Lisæter, K., G. Evensen, and S. Laxon, 2007: Assimilating synthetic CryoSat sea ice thickness in a coupled ice-ocean model. J. Geophys. Res., 112, C07023, https://doi.org/ 10.1029/2006JC003786.

Massonnet, F., T. Fichefet, H. Goosse, M. Vancoppenolle, P. Mathiot, and C. König Beatty, 2011: On the influence of model physics on simulations of Arctic and Antarctic sea ice. Cryosphere, 5, 687-699, https://doi.org/10.5194/tc-5-687-2011.

,-- , and — 2015: Prospects for improved seasonal Arctic sea ice predictions from multivariate data assimilation. Ocean Modell., 88, 16-25, https://doi.org/10.1016/j.ocemod.2014.12.013.

Menemenlis, D., J.-M. Campin, P. Heimbach, C. Hill, T. Lee, A. Nguyen, M. Schodlok, and H. Zhang, 2008: ECCO2: High resolution global ocean and sea ice data synthesis. Mercator Ocean Quarterly Newsletter, No. 31, Mercator Océan, Ramonville-Saint-Agne, France, 13-21.

Miller, P. A., S. W. Laxon, D. L. Feltham, and D. J. Cresswell, 2006 Optimization of a sea ice model using basinwide observations of Arctic sea ice thickness, extent, and velocity. J. Climate, 19, 1089-1108, https://doi.org/10.1175/JCLI3648.1.

Murashkin, D., G. Spreen, M. Huntemann, and W. Dierking, 2018: Method for detection of leads from Sentinel-1 SAR images. Ann. Glaciol., 59, 124-136, https://doi.org/10.1017/aog.2018.6.

Neumaier, A., 1998: Solving ill-conditioned and singular linear systems: A tutorial on regularization. SIAM Rev., 40, 636-666, https://doi.org/10.1137/S0036144597321909.

Peng, G., W. Meier, D. Scott, and M. Savoie, 2013: A long-term and reproducible passive microwave sea ice concentration data record for climate studies and monitoring. Earth Syst. Sci. Data, 5, 311-318, https://doi.org/10.5194/essd-5-311-2013.

Posey, P., and Coauthors, 2015: Assimilating high horizontal resolution sea ice concentration data into the US Navy's ice forecast systems: Arctic Cap Nowcast/Forecast System (ACNFS) and the Global Ocean Forecast System (GOFS 3.1). Cryosphere, 9, 1735-1745, https://doi.org/10.5194/tcd9-2339-2015.
Pritchard, R. S., 2005: Stability of sea ice dynamics models: Viscous-plastic rheology, replacement closure, and tensile cutoff. J. Geophys. Res., 110, C12010, https://doi.org/ 10.1029/2003JC001875.

Rampal, P., S. Bouillon, E. Ólason, and M. Morlighem, 2016: neXtSIM: A new Lagrangian sea ice model. Cryosphere, 10, 1055-1073, https://doi.org/10.5194/tc-10-1055-2016.

Saad, Y., and M. H. Schultz, 1986: GMRES: A generalized minimal residual algorithm for solving nonsymmetric linear systems. SIAM J. Sci. Stat. Comput., 7, 856-869, https://doi.org/10.1137/ 0907058.

Sakov, P., F. Counillon, L. Bertino, K. Lisæter, P. Oke, and A. Korablev, 2012: TOPAZ4: An ocean-sea ice data assimilation system for the North Atlantic and Arctic. Ocean Sci., 8 , 633-656, https://doi.org/10.5194/os-8-633-2012.

Schulkes, R., 1996: Asymptotic stability of the viscous-plastic sea ice rheology. J. Phys. Oceanogr., 26, 279-283, https://doi.org/ 10.1175/1520-0485(1996)026<0279:ASOTVS > 2.0.CO;2.

Schwegmann, S., C. Haas, C. Fowler, and R. Gerdes, 2011: A comparison of satellite-derived sea-ice motion with driftingbuoy data in the Weddell Sea, Antarctica. Ann. Glaciol., 52, 103-110, https://doi.org/10.3189/172756411795931813.

Steele, M., J. H. Morison, and N. Untersteiner, 1989: The partition of air-ice-ocean momentum exchange as a function of ice concentration, floe size, and draft. J. Geophys. Res., 94, 12739 12 750, https://doi.org/10.1029/JC094iC09p12739.

Steiner, N., 2001: Introduction of variable drag coefficients into sea-ice models. Ann. Glaciol., 33, 181-186, https://doi.org/ 10.3189/172756401781818149.

Sumata, H., R. Kwok, R. Gerdes, F. Kauker, and M. Karcher, 2015: Uncertainty of Arctic summer ice drift assessed by highresolution SAR data. J. Geophys. Res. Oceans, 120, 52855301, https://doi.org/10.1002/2015JC010810.

Tilling, R. L., A. Ridout, and A. Shepherd, 2018: Estimating Arctic sea ice thickness and volume using CryoSat-2 radar altimeter data. Adv. Space Res., 62, 1203-1225, https://doi.org/10.1016/ j.asr.2017.10.051.

Timco, G., and W. Weeks, 2010: A review of the engineering properties of sea ice. Cold Reg. Sci. Technol., 60, 107-129, https://doi.org/10.1016/j.coldregions.2009.10.003.

Timoshenko, S., and J. Goodier, 1951: Theory of Elasticity. McGraw-Hill, 567 pp.

Toyota, T., and N. Kimura, 2018: An examination of the sea ice rheology for seasonal ice zones based on ice drift and thickness observations. J. Geophys. Res. Oceans, 123, 1406-1428, https:// doi.org/10.1002/2017JC013627.

Tremblay, L., and M. Hakakian, 2006: Estimating the sea ice compressive strength from satellite-derived sea ice drift and NCEP reanalysis data. J. Phys. Oceanogr., 36, 2165-2172, https:// doi.org/10.1175/JPO2954.1.

Tschudi, M., W. Meier, J. Stewart, C. Fowler, and J. Maslanik, 2019: Polar Pathfinder daily $25 \mathrm{~km}$ EASE-Grid sea ice motion vectors, version 4 dataset 0116. NASA National Snow and Ice Data Center Distributed Active Archive Center, accessed 17 March 2019, https://doi.org/10.5067/INAWUWO7QH7B.

Vancoppenolle, M., T. Fichefet, H. Goosse, S. Bouillon, G. Madec, and M. A. M. Maqueda, 2009: Simulating the mass balance and salinity of Arctic and Antarctic sea ice. 1. Model description and validation. Ocean Modell., 27, 33-53, https://doi.org/10.1016/ j.ocemod.2008.10.005.

Wang, K., J. Debernard, A. K. Sperrevik, P. E. Isachsen, and T. Lavergne, 2013: A combined optimal interpolation and nudging scheme to assimilate OSISAF sea-ice concentration 
into ROMS. Ann. Glaciol., 54, 8-12, https://doi.org/10.3189/ 2013AoG62A138.

Weeks, W. F., and A. Assur, 1967: The mechanical properties of sea ice. Cold Regions Research and Engineering Lab Tech. Rep., 96 pp. Yaremchuk, M., T. Townsend, G. Panteleev, D. Hebert, and R. Allard, 2019: Advancing short-term forecasts of ice conditions in the Beaufort Sea. J. Geophys. Res. Oceans, 124, 807820, https://doi.org/10.1029/2018JC014581.

Zakhvatkina, N. Y., V. Y. Alexandrov, O. M. Johannessen, S. Sandven, and I. Y. Frolov, 2013: Classification of sea ice types in ENVISAT synthetic aperture radar images. IEEE
Trans. Geosci. Remote Sens., 51, 2587-2600, https://doi.org/ 10.1109/TGRS.2012.2212445.

, A. Korosov, S. Muckenhuber, S. Sandven, and M. Babiker, 2017: Operational algorithm for ice-water classification on dual-polarized RadarSat-2 images. Cryosphere, 11, 33-46, https://doi.org/10.5194/tc-11-33-2017.

Zhang, J., and D. Rothrock, 2003: Modeling global sea ice with a thickness and enthalpy distribution model in generalized curvilinear coordinates. Mon. Wea. Rev., 131, 845-861, https://doi.org/10.1175/1520-0493(2003)131<0845:MGSIWA> 2.0.CO;2. 